\title{
Modified Reynolds Equation for Steady Flow Through a Curved Pipe
}

\author{
A. Ghosh(D, V. A. Kozlov and S. A. Nazarov \\ Communicated by G. P. Galdi
}

\begin{abstract}
A Reynolds equation governing the steady flow of a fluid through a curvilinear, narrow tube, with its derivation from Navier-Stokes equations through asymptotic methods is presented. The channel considered may have a rather large curvature and torsion. Approximations of the velocity and the pressure of the fluid inside the channel are constructed by artificially imposing appropriate boundary conditions at the inlet and the outlet. A justification for the approximations is provided along with a comparison with a simpler case.
\end{abstract}

\section{Introduction}

Problems on fluid flow through narrow tubes describe a wide range of processes occuring in nature, including biological or non-biological systems, as well as man-made architectural and industrial products. These processes have varying scales, for example consider oil pipelines on one hand and circulatory systems in vertebrates on the other. Despite the fact that the Reynolds equation was first derived over 130 years ago [16], the asymptotic analysis of thin flows still attracts interest from researchers worldwide, see for example $[2,13,14]$ and references therein. The result of applying a well-known dimension reduction procedure providing a meaningful transition from the three-dimensional problem to its one-dimensional model, remains the same though: ordinary second-order differential equation in its divergence form on the axis of the channel. The scalar coefficient of the differential operator in this equation (the Reynolds coefficient) is determined based on the classical Poiseuille-Reynolds asymptotic ansatz and correlates to the normal section of the channel, either smoothly changing or rapidly oscillating; see [12] and [1] respectively. What is remarkable is the fact that in the limit equation, the above mentioned coefficient is independent of the curvature and twist of the central axis of the channel in the ambient three-dimensional space, see for example [10]. We emphasize that a distortion of the Poiseuille flow, smoothly changing along the channel axis, occurs only near localized shape perturbations such as the ends of the thin channel, near its kinks or other areas with large curvatures as well as at the nodes of junctions of the thin channels, see $[10,12-14]$ etc.

In [10], the complete asymptotic series is constructed for the solution of the Navier-Stokes problem in a thin, smoothly curved channel having curvature and twist of order 1 with respect to the relative thickness and with a constant cross-section of small diameter. This series consists of terms of the regular type (smooth functions on the channel axis multiplied by vector functions described in stretched coordinates on the cross-section) and boundary layer type terms localized near the ends of the channel (exponentially decaying solutions of the linear Stokes problem in semi-infinite cylinders). Justification of the asymptotics is carried out under the implicitly stated restriction: the flux (surface integral of velocity) through a crosssection is $O\left(\varepsilon^{2}\right)$, where $\varepsilon$ is the small aspect ratio of the channel.

In this paper, we consider nonlinear Navier-Stokes equations as well but supply them with mixed boundary conditions: a no-slip condition is assigned on the lateral boundary of the channel, the flux

S. A. Nazarov acknowledges the support from Russian Foundation of Basic Research, Grant 18-01-00325. 
through the cross-section is given at one end of the channel while the hydrostatic pressure is prescribed at the other end. A discussion about these conditions is provided in this section while their exact formulation is given in Sect. 5 .

We consider the problem from a completely different point of view as compared to that in [10]: we refuse to construct higher-order asymptotic terms, including the boundary layer terms, and instead apply asymptotic analysis to find a modified Reynolds coefficient in the standard Reynolds equation so that a solution of the new equation gives an approximation of improved accuracy for the velocity and pressure fields. Namely, the modified Reynolds coefficient is defined through the formulas (39) and (45) where a scalar function $\beta$ appears (see (14)), that depends on the channel curvature and the small aspect ratio $h$ of the channel. The main terms of the asymptotic ansatz are modified accordingly. This means that the Poiseuille flow is also modified taking the channel curvature into account.

Our proposed simple modification of the well known one-dimensional model (constructed by solving a Dirichlet scalar problem on a stretched cross-section of the channel) provide at least two advantages. Firstly, it remains suitable even in the case of high curvature of order $h^{-1+\delta}$ for any positive parameter $\delta$. Notice that earlier, for example in [10], these geometric extremes were never considered. Secondly, in the case of curvatures of order $1=h^{0}$, a solution of a single scalar equation with the introduced modification, furnishes at least two leading asymptotic terms of the regular type simultaneously.

If the first improvement is rather unexpected (at least for the authors), the second one can be traced to other problems in mechanics and physics: for example, a system of two-dimensional differential equations describing the deformation of a thin, elastic thee-dimensional shell. However, the improvement in the accuracy of the approximation is limited by several circumstances. Primary among them, the boundary layer phenomenon, which, having a strictly spatial structure, cannot be described in the framework of one-dimensional models.

In most issues related to thin flows, truncation of a domain on which the boundary problem is posed, is purely a mathematical convention. However, the choice of artificial boundary conditions on the truncation surfaces that do not affect the main asymptotic terms, plays a crucial role in constructing the asymptotic correction terms. See [3] for comparision where boundary layers at the inlet and outlet are considered while studying the asymptotic solution for micropolar flow through a two-dimensional curvilinear channel. Usually, a profile of the three-dimensional velocity vector is prescribed at the inlet, whereas the boundary condition in the one-dimensional model only incorporates its averaged characteristics, that is, the flux. The above mentioned profile is prescribed as arbitrary or is fixed without a proper justification - the authors are unaware of studies taking into account a mechanism to push fluid into the channel, for instance, as in the functioning of a heart with its valve, the action of an injector, the influence of the shape of a piston, etc. In this way, most of the one-dimensional models are applied away from the "perturbing" objects, where the influence of the boundary layers prescribing rapid variability of the velocity vector and pressure, is negligible or simply absent. This observation prompts us to abandon the study of the boundary layers and accept the "well prepared" boundary conditions at the artificially introduced ends of the finite channel: the velocity vector profile at the ends appear to be prescribed by the asymptotic ansatz in the one-dimensional model which reflects as only scalar quantities at the ends - the flux and the averaged pressure, while the corresponding profiles are reconstructed by the asymptotic procedure itself.

When justifying the correctly formulated boundary value problem (with the fixed profiles of velocities and pressure at the ends), the influence of the boundary layers is reduced by means of a cut-off function, which requires special processing using different lemmas on the divergence equation (see Lemma 1 and Lemma 2). To facilitate the demonstration, we first obtain error estimates for asymptotic residues in the case of the linear Stokes equations and only then we process the convective term. Thus, we establish a limit on the Reynolds number, allowing to linearize the Navier-Stokes equation and construct its onedimensional model. 


\subsection{Nondimensionalization of the Problem}

Let us consider a curvilinear pipe of length $L$ with varying channel diameter and non-circular cross-section. Let us denote the mean radius by $H$ which satisfies $H=L h$. We assume that the pipe is narrow or in other words, $h \ll 1$ is a small, dimensionless, positive parameter. Let us denote the steady state velocity and the kinematic pressure of the flowing fluid by $\mathbf{V}$ and $P$ respectively. These satisfy the Navier-Stokes system

$$
\begin{aligned}
-\nu \Delta_{\mathbf{X}} \mathbf{V}+ & \left(\mathbf{V} \cdot \nabla_{\mathbf{X}}\right) \mathbf{V}+\nabla_{\mathbf{X}} P=0, \\
& -\nabla_{\mathbf{X}} \cdot \mathbf{V}=0,
\end{aligned}
$$

complemented by suitable boundary conditions and where $\nu$ is the kinematic viscosity of the fluid flowing through the tube and $\mathbf{X}$ signifies the position vector in suitable units of length.

Let $F$ be the flux through a cross-section. We introduce new dimensionless variables for our problem:

$$
\mathbf{x}=\frac{1}{L} \mathbf{X}, \quad \mathbf{v}=\frac{H^{2}}{F} \mathbf{V} \quad \text { and } \quad p=\frac{H^{2} L}{\nu F} P .
$$

In terms of the new dimensionless quantities and the Reynolds number $\operatorname{Re}:=\frac{F L}{\nu H^{2}}$, the Navier-Stokes equations take the form

$$
-\Delta_{\mathbf{x}} \mathbf{v}+\operatorname{Re}\left(\mathbf{v} \cdot \nabla_{\mathbf{x}}\right) \mathbf{v}+\nabla_{\mathbf{x}} p=0
$$

For the article, we assume a small Reynolds number Re so that we are able to rigorously discard the convective term.

\subsection{Formulation of the Problem}

Let $\mathbf{c}:[0,1] \rightarrow \mathbb{R}^{3}$ denote the arc-length parameterized centre curve for the pipe segment in consideration with $s \in[0,1]$ denoting the arc-length parameter. The derivatives of the vector function $\mathbf{c}$ are represented by the corresponding number of prime symbols ' appended to it. Let $h R>0$ give the distance of the interior boundary of the pipe from $\mathbf{c}(s)$ along a direction which is perpendicular to the centre curve at $s$.

Let us consider the liquid domain, its longitudinal surface and its transversal cross-sections respectively:

$$
\begin{aligned}
\Omega^{h} & =\{\mathbf{x}(r, \theta, s): 0 \leq r<h R(\theta, s), \theta \in[0,2 \pi), s \in(0,1)\}, \\
\Sigma^{h} & =\left\{\mathbf{x} \in \partial \Omega^{h}: s \in(0,1)\right\}, \\
\omega(s) & =\left\{\mathbf{c}(s)+\eta \mathbf{e}_{1}(\theta, s): \eta=h^{-1} r, 0 \leq r<h R(\theta, s), \theta \in[0,2 \pi)\right\} .
\end{aligned}
$$

The region $\Omega^{h}$ is assumed to be locally Lipschitz and each transversal cross-section $\omega(s)$ to be a star domain for every $s$. The radial unit vector function $\mathbf{e}_{1}$ is defined in the next section. For a cross-section with a fixed $s$, the pair $(r, \theta)$ represent the polar coordinate system.

The goal is to find an asymptotic approximation of the solution of the linearized Stokes equations

$$
\begin{aligned}
-\Delta_{\mathbf{x}} \mathbf{v}^{h}+\nabla_{\mathbf{x}} p^{h}=\mathbf{0} & \text { in } \quad \Omega^{h}, \\
-\nabla_{\mathbf{x}} \cdot \mathbf{v}^{h}=0 & \text { in } \quad \Omega^{h}, \\
\mathbf{v}^{h}=\mathbf{0} & \text { on } \quad \Sigma^{h},
\end{aligned}
$$

supplemented with appropriate boundary conditions at the sections $s=0$ and $s=1$. We ignore the convective term in the formal analysis. 


\subsection{Results}

The primary highlight of this article is the derivation of a Reynolds type equation for flow through a curved pipe

$$
-\partial_{s}\left(G(s) \partial_{s} p^{0}(s)\right)=0, \quad s \in(0,1),
$$

with a new formula for the Reynolds coefficient. The coefficient $G$, defined as

$$
G(s):=2 \int_{\omega(s)} \Psi(\eta, \theta, s) \eta \mathrm{d} \eta \mathrm{d} \theta,
$$

depends on the geometry of the pipe and $p^{0}$ is the leading term in the formal asymptotic expansion of $p^{h}$ as stated in (36). Defining $\beta(\eta, \theta, s)$ as the scale factor corresponding to the longitudinal parameter $s$ and $\nabla_{\ddagger}$ as the gradient operator on the plane $\omega(s)$, the function $\Psi$ is obtained as the solution of

$$
-\nabla_{\ddagger} \cdot \beta(\eta, \theta, s) \nabla_{\ddagger} \Psi(\eta, \theta, s)=2 \quad \text { in } \quad \omega(s), \quad \Psi(\eta, \theta, s)=0 \quad \text { on } \quad \partial \omega(s) .
$$

The function $\beta$ plays a central role in this work as it is mostly through this function, the influence of the curvature is manifested in the resulting model equations. It is related to the curvature by the formula

$$
\beta(\eta, \theta, s)=1-h \eta \mathbf{c}^{\prime \prime}(s) \cdot \mathbf{e}_{1}(\theta, s) .
$$

Note that in the absence of curvature, the scale function $\beta$ is identically equal to 1 everywhere. Also, in the case of curvature of order 1 with respect to $h$, we have $\beta=1+O(h)$ and hence we arrive at the usual Reynolds equation.

The new Reynolds equation takes into consideration a relatively wide range of curvature as well as variation of the diameter of the pipe as characterized by the following restrictions:

$$
\begin{aligned}
& \quad\left|\mathbf{c}^{\prime \prime \prime}(s)\right| \leq c h^{-2+2 \delta}, \quad\left|\mathbf{c}^{\prime \prime \prime \prime}(s)\right| \leq c h^{-3+3 \delta}, \\
& \quad\left|\partial_{s} R(\theta, s)\right| \leq c h^{-1+2 \delta} \quad \text { and } \quad\left|\partial_{s}^{2} R(\theta, s)\right| \leq c h^{-2+3 \delta}
\end{aligned}
$$

for some positive $\delta$. In particular, the first inequality implies the following bound for the curvature:

$$
\left|\mathbf{c}^{\prime \prime}(s)\right| \leq c h^{-1+\delta} .
$$

Note that this means that the pipe cannot curve into itself as long as $\delta>0$. The inequalities in (5) can be interpreted as follows: a differentiation with respect to the longitudinal parameter $s$ can at most be of order $O\left(h^{-1+\delta}\right)$. Thus while allowing for high curvatures and variations of the surface of the pipe, the restrictions do not allow kinks or sharp bends of the pipe which would need to be treated separately.

Our approach in this article is to treat the geometrical quantities in (5) as separate parameters in the beginning and choosing the optimal orders by the end of the analysis. The new equation covers in particular the case of smooth curvature (order 1 with respect to $h$ ) and the case of nearly constant radius (order $h$ ) of the pipe as well which is achieved by replacing the bounds in (5) by a constant independent of $h$.

Based on the new Reynolds equation, under the introduced assumptions and provided the appropriate boundary conditions, we proceed to construct an approximation $\left\{\mathbf{v}^{h}, \mathbb{p}^{h}\right\}$ for the solution $\left\{\mathbf{v}^{h}, p^{h}\right\}$ of $(1)-$ (3) in the form

$$
\begin{aligned}
& \mathbb{P}^{h}(\mathbf{x}) \approx h^{-3} p^{0}(s), \\
& \mathbf{v}^{h}(\mathbf{x}) \approx h^{-1} v_{3}^{1}(\eta, \theta, s) \mathbf{c}^{\prime}(s)+\mathbf{v}_{\ddagger}^{2}(\eta, \theta, s) .
\end{aligned}
$$

Accordingly, we obtain the representation

$$
\mathbf{v}^{h}=\mathbf{v}^{h}+\mathbf{v}_{r e m}^{h} \quad \text { and } \quad p^{h}=p^{h}+p_{r e m}^{h} .
$$

We finally prove that in the linear problem and under certain assumption on the Reynolds number in the non-linear case, the error terms $\left\{\mathbf{v}_{r e m}^{h}, p_{\text {rem }}^{h}\right\}$ in (6) admit the bounds

$$
h\left\|\nabla_{\mathbf{x}} \mathbf{v}_{r e m}^{h}\right\|+\left\|\mathbf{v}_{r e m}^{h}\right\|+h^{2}\left\|p_{r e m}^{h}-\bar{p}_{r e m}^{h}\right\| \leq c h^{\delta}
$$




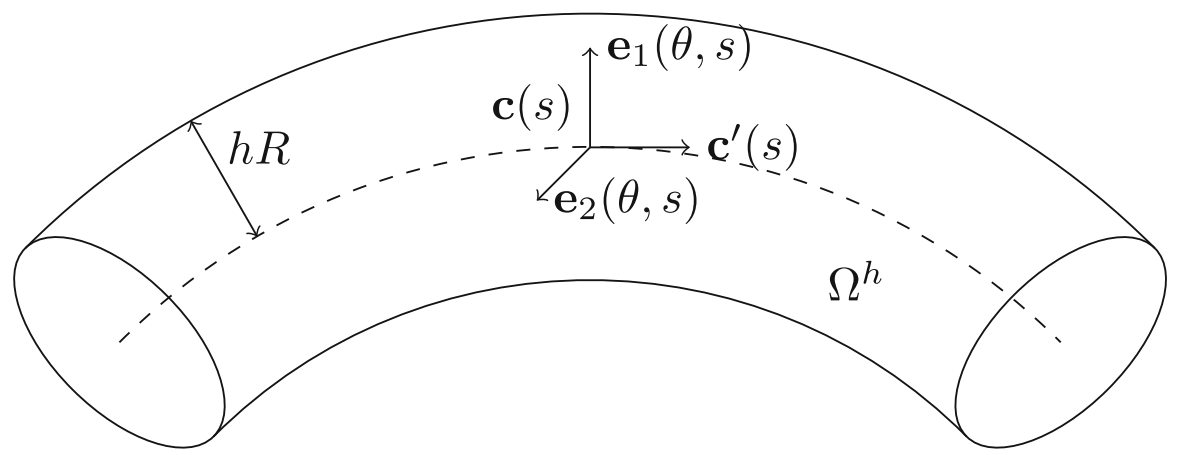

FIG. 1. The curvilinear coordinate frame $\left\{\mathbf{e}_{1}, \mathbf{e}_{2}, \mathbf{c}^{\prime}\right\}$ depicted at the point $\mathbf{c}(s)$ on the centre curve in the domain $\Omega^{h}$.

whereas $\left\{\boldsymbol{v}^{h}, \mathbb{p}^{h}\right\}$ admit the relation

$$
h\left\|\nabla_{\mathbf{x}} \mathbf{v}^{h}\right\|+\left\|\mathbf{v}^{h}\right\|+h^{2}\left\|p^{h}-\overline{\mathbb{p}}^{h}\right\| \leq c h^{0}
$$

thereby justifying the approximate solution for any positive $\delta$.

\section{Geometry and Notations}

The ambient three dimensional space is taken to have a canonical Cartesian coordinate system. The initial direction of the curve is assumed to be along the third coordinate axis, i.e., $\mathbf{c}^{\prime}(0)=(0,0,1)^{T}$. The vector quantities for the problem are described using the coordinate frame consisting of the triplet $\left\{\mathbf{e}_{1}(\theta, s), \mathbf{e}_{2}(\theta, s), \mathbf{c}^{\prime}(s)\right\}$, depicted in Fig. 1, where $\mathbf{e}_{i}$ are obtained by solving the Cauchy problem

$$
\partial_{s} \mathbf{e}_{i}(\theta, s)=-\left(\mathbf{c}^{\prime \prime}(s) \cdot \mathbf{e}_{i}(\theta, s)\right) \mathbf{c}^{\prime}(s)
$$

with the initial conditions

$$
\mathbf{e}_{1}(\theta, 0)=(\cos \theta, \sin \theta, 0)^{T} \text { and } \mathbf{e}_{2}(\theta, 0)=(-\sin \theta, \cos \theta, 0)^{T} .
$$

Additionally, they also satisfy

$$
\partial_{\theta} \mathbf{e}_{1}(\theta, s)=\mathbf{e}_{2}(\theta, s) \text { and } \partial_{\theta} \mathbf{e}_{2}(\theta, s)=-\mathbf{e}_{1}(\theta, s) .
$$

Remark 1. The frame $\left\{\mathbf{e}_{1}(\theta, s), \mathbf{e}_{2}(\theta, s), \mathbf{c}^{\prime}(s)\right\}$ is an orthonormal frame of reference. As opposed to the Frenet-Serret frame, it is well defined even in the curvature-free segments of the pipe.

The parameter $\theta \in[0,2 \pi)$ signifies the direction from a point on $\mathbf{c}$, along the plane perpendicular to $\mathbf{c}^{\prime}$ at that point, with respect to some reference direction. Throughout this article, vectors are denoted in bold while their components along $\mathbf{e}_{1}, \mathbf{e}_{2}$ and $\mathbf{c}^{\prime}$ are given by the corresponding letter with subscripts 1,2 and 3 respectively.

With this frame, we have new curvilinear coordinates $\{r, \theta, s\}$ which are related to the Cartesian coordinates by

$$
\mathbf{x}(r, \theta, s)=\mathbf{c}(s)+r \mathbf{e}_{1}(\theta, s), \quad 0 \leq r \leq h R(\theta, s) .
$$

Note that in the absence of curvature, $\{r, \theta, s\}$ are cylindrical coordinates for the straight tube.

Clearly, $R$ must be positive and sufficiently smooth and we define

$$
\gamma:=\max _{(\theta, s) \in[0,2 \pi) \times[0,1]}\left|\partial_{s} R(\theta, s)\right|
$$

and

$$
\gamma^{*}:=\max _{(\theta, s) \in[0,2 \pi) \times[0,1]}\left|\partial_{s}^{2} R(\theta, s)\right| .
$$


Additionally, we also define

$$
\lambda:=\max _{s \in[0,1]}\left|h \mathbf{c}^{\prime \prime \prime}(s)\right|
$$

along with

$$
\lambda^{*}:=\max _{s \in[0,1]}\left|h \mathbf{c}^{\prime \prime \prime \prime}(s)\right| .
$$

For arc-length parametrization, the scalar product $\mathbf{c}^{\prime \prime}(s) \cdot \mathbf{c}^{\prime}(s)$ vanishes for all $s$. Differentiating the scalar product with respect to $s$ and with $\lambda$ as defined above, we get

$$
\left|\mathbf{c}^{\prime \prime}(s)\right|=\sqrt{\left|\mathbf{c}^{\prime \prime \prime}(s) \cdot \mathbf{c}^{\prime}(s)\right|} \leq h^{-1 / 2} \lambda^{1 / 2} \quad \forall s \in[0,1] .
$$

This means imposing bounds on the third derivative $\mathbf{c}^{\prime \prime \prime}$ results in a corresponding upper bound for the curvature $\left|\mathbf{c}^{\prime \prime}\right|$ thereby eliminating the need for introduction of an additional parameter for it. Here we recall that the prime symbol signifies a derivative with respect to the only variable in the argument of a function.

Let $\nabla$. be the two dimensional gradient operator on a cross-section, i.e.,

$$
\nabla_{\bullet}:=\mathbf{e}_{1} \partial_{r}+\frac{1}{r} \mathbf{e}_{2} \partial_{\theta} .
$$

Let $\nabla_{\ddagger}$ and $\Delta_{\ddagger}$ denote the components of the gradient operator and the Laplacian respectively, on a cross-section in terms of the scaled parameters $\eta:=h^{-1} r$ and $\theta$, i.e.,

$$
\nabla_{\ddagger}:=\mathbf{e}_{1} \partial_{\eta}+\frac{1}{\eta} \mathbf{e}_{2} \partial_{\theta}=h \nabla \bullet \quad \text { and } \quad \Delta_{\ddagger}:=\nabla_{\ddagger} \cdot \nabla_{\ddagger}=\partial_{\eta}^{2}+\frac{1}{\eta} \partial_{\eta}+\frac{1}{\eta^{2}} \partial_{\theta}^{2} .
$$

Then, we have

$$
\nabla_{\mathbf{x}}=\nabla_{\bullet}+\beta^{-1} \mathbf{c}^{\prime} \partial_{s}=h^{-1} \nabla_{\ddagger}+\beta^{-1} \mathbf{c}^{\prime} \partial_{s},
$$

and

$$
\begin{aligned}
\Delta_{\mathbf{x}} & =h^{-2} \Delta_{\ddagger}-h^{-1} \beta^{-1} \mathbf{c}^{\prime \prime} \cdot \nabla_{\ddagger}+\beta^{-2} \partial_{s}^{2}+h \beta^{-3} \eta \mathbf{c}^{\prime \prime \prime} \cdot \mathbf{e}_{1} \partial_{s} \\
& =h^{-2} \beta^{-1} \nabla_{\ddagger} \cdot \beta \nabla_{\ddagger}+\beta^{-1} \partial_{s} \beta^{-1} \partial_{s}
\end{aligned}
$$

where we have introduced the scale factor

$$
\beta(r, \theta, s):=\left|\partial_{s} \mathbf{x}(r, \theta, s)\right|=1-r \mathbf{c}^{\prime \prime}(s) \cdot \mathbf{e}_{1}(\theta, s)=1-h \eta \mathbf{c}^{\prime \prime}(s) \cdot \mathbf{e}_{1}(\theta, s)
$$

corresponding to the parameter $s$ and used the fact that $\nabla \cdot \beta=h^{-1} \nabla_{\ddagger} \beta=-\mathbf{c}^{\prime \prime}$. Then due to (11), (12) and (13), we have

$$
\left|\partial_{s} \beta\right| \leq c \lambda \quad \text { and } \quad\left|\partial_{s}^{2} \beta\right| \leq c\left(\lambda^{*}+h^{-1 / 2} \lambda^{3 / 2}\right)
$$

As has been mentioned, the restriction on the curvature such that $h \mathbf{c}^{\prime \prime}(s) \cdot \mathbf{e}_{1}(\theta, s)<R(\theta, s)^{-1}$ for all $(\theta, s) \in[0,2 \pi) \times[0,1]$ eliminates the possibility of the pipe curving into itself. Moreover, to ensure the validity of the asymptotic procedure followed in this article, we must additionally assume

$$
\lambda=o\left(h^{-1}\right) .
$$

For integration over the cross sections, we have the area element

$$
\mathrm{d} \sigma(r, \theta)=r \mathrm{~d} r \mathrm{~d} \theta \text {. }
$$

On the other hand, the volume element is given in the new coordinates as

$$
\mathrm{d} \mathbf{x}(r, \theta, s)=\beta(r, \theta, s) \mathrm{d} \sigma(r, \theta) \mathrm{d} s .
$$

Let us denote the velocity vector component wise as $\mathbf{v}^{h}=v_{1}^{h} \mathbf{e}_{1}+v_{2}^{h} \mathbf{e}_{2}+v_{3}^{h} \mathbf{c}^{\prime}$. The components of the quantities in (1) along any cross-section $\omega(s)$ of the pipe satisfy

$$
\begin{aligned}
& -h^{-2} \beta^{-1} \nabla_{\ddagger} \cdot \beta \nabla_{\ddagger} \mathbf{v}_{\ddagger}^{h}+h^{-1} \nabla_{\ddagger} p^{h}+\beta^{-2} \mathbf{c}^{\prime \prime} \mathbf{c}^{\prime \prime} \cdot \mathbf{v}_{\ddagger}^{h}-\mathbf{c}_{\ddagger}^{\prime \prime \prime} \beta^{-2} v_{3}^{h}-\mathbf{c}^{\prime \prime} \beta^{-2} \partial_{s} v_{3}^{h} \\
& -\mathbf{c}^{\prime \prime} \beta^{-1} \partial_{s}\left(\beta^{-1} v_{3}^{h}\right)-\sum_{i=1,2} \mathbf{e}_{i} \beta^{-1} \partial_{s}\left(\beta^{-1} \partial_{s} v_{i}^{h}\right)=\mathbf{0}_{\ddagger} \quad \text { in } \quad \Omega^{h} .
\end{aligned}
$$


On the other hand, (1) results in the following equation for the direction along the length of the pipe:

$$
\begin{gathered}
-h^{-2} \beta^{-1} \nabla_{\ddagger} \cdot \beta \nabla_{\ddagger} v_{3}^{h}+\beta^{-2} \mathbf{c}^{\prime \prime \prime} \cdot \mathbf{v}_{\ddagger}^{h}+\sum_{i=1,2} \mathbf{c}^{\prime \prime} \cdot \mathbf{e}_{i} \beta^{-1} \partial_{s}\left(\beta^{-1} v_{i}^{h}\right) \\
-\beta^{-2} \mathbf{c}^{\prime \prime \prime} \cdot \mathbf{c}^{\prime} v_{3}^{h}-\beta^{-1} \partial_{s}\left(\beta^{-1} \partial_{s} v_{3}^{h}\right)+\beta^{-1} \partial_{s} p^{h}=0 \quad \text { in } \quad \Omega^{h} .
\end{gathered}
$$

Finally, the divergence equation (2) can be reformulated as

$$
-h^{-1} \beta^{-1} \nabla_{\ddagger} \cdot \beta \mathbf{v}_{\ddagger}^{h}-\beta^{-1} \partial_{s} v_{3}^{h}=0 \quad \text { in } \quad \Omega^{h} .
$$

In the above and henceforth, $\ddagger$ in the subscript of the vector symbols denote their respective projections onto the cross-sectional plane.

In order to ensure uniqueness of the asymptotic solution of the equations, we intend to impose additional artificial conditions at the ends of the pipe. We shall argue in the next sections that a prescribed flux at the inlet and an ambient (possibly atmospheric) pressure condition at the outlet are sufficient for our purpose. On the lateral boundary, we assume no-slip conditions.

\section{Model Problems and Estimates}

In this section, we present the estimates related to some model problems that we rely upon in the asymptotic procedure. We use similar notations for function spaces as in [17], which include standard notations for Sobolev spaces. In particular, the function spaces denoted by bold letters represent the corresponding space of vector/tensor valued functions of the appropriate dimension.

\subsection{Stokes System}

We first consider a modified Stokes problem on the two dimensional domain $\omega(s)$. We present the relevant estimates in the theorem that follows.

Theorem 1. Let there be given $\mathbf{f} \in \mathbf{H}^{-1}(\omega(s)), g \in L^{2}(\omega(s))$ and $\mathbf{h} \in \mathbf{H}^{1 / 2}(\partial \omega(s))$ satisfying the compatibility condition,

$$
\int_{\omega(s)} \beta g \mathrm{~d} \sigma(\eta, \theta)+\int_{0}^{2 \pi} \beta \mathbf{h} \cdot\left(R \mathbf{e}_{1}-\left(\partial_{\theta} R\right) \mathbf{e}_{2}\right) \mathrm{d} \theta=0 .
$$

Then there exist a unique $\mathbf{u} \in \mathbf{H}^{1}(\omega(s))$ and a unique $q \in L^{2}(\omega(s))$ up to a constant that solve the two-dimensional modified Stokes problem

$$
\begin{gathered}
-\beta^{-1} \nabla_{\ddagger} \cdot \beta \nabla_{\ddagger} \mathbf{u}+\nabla_{\ddagger} q=\mathbf{f}, \quad-\beta^{-1} \nabla_{\ddagger} \cdot \beta \mathbf{u}=g \quad \text { in } \quad \omega(s), \\
\mathbf{u}=\mathbf{h} \quad \text { on } \quad \partial \omega(s) .
\end{gathered}
$$

The solutions admit the estimate

$$
\begin{aligned}
& \left\|\nabla_{\ddagger} \mathbf{u}\right\|_{\mathbf{L}^{2}(\omega(s))}+\|\mathbf{u}\|_{\mathbf{L}^{2}(\omega(s))}+\|q-\bar{q}\|_{L^{2}(\omega(s))} \\
& \quad \leq c\left(\|\mathbf{f}\|_{\mathbf{H}^{-1}(\omega(s))}+\|g\|_{L^{2}(\omega(s))}+\|\mathbf{h}\|_{\mathbf{H}^{1 / 2}(\partial \omega(s))}\right),
\end{aligned}
$$

where $\bar{q}$ is $q$ averaged over $\omega(s)$.

Furthermore, if $\mathbf{f} \in \mathbf{L}^{2}(\omega(s)), g \in H^{1}(\omega(s))$ and $\mathbf{h} \in \mathbf{H}^{3 / 2}(\partial \omega(s))$, then $\mathbf{u} \in \mathbf{H}^{2}(\omega(s))$ and $q \in$ $H^{1}(\omega(s))$ satisfy

$$
\begin{aligned}
& \left\|\nabla_{\ddagger} \nabla_{\ddagger} \mathbf{u}\right\|_{\mathbf{L}^{2}(\omega(s))}+\left\|\nabla_{\ddagger} q\right\|_{\mathbf{L}^{2}(\omega(s))} \\
& \quad \leq c\left(\|\mathbf{f}\|_{\mathbf{L}^{2}(\omega(s))}+\|g\|_{H^{1}(\omega(s))}+\|\mathbf{h}\|_{\mathbf{H}^{3 / 2}(\partial \omega(s))}\right) .
\end{aligned}
$$


Proof. We accept (22) without proof as it is a standard estimate for generalised Stokes systems, see e.g. [5] that can be applied to this case owing to the boundedness of the parameter $\beta$. In order to obtain (23), we rewrite $(21)$ as

$$
\begin{gathered}
-\nabla_{\ddagger} \cdot \nabla_{\ddagger} \mathbf{u}+\nabla_{\ddagger} q=\mathbf{f}+h \beta^{-1} \mathbf{c}^{\prime \prime} \cdot \nabla_{\ddagger} \mathbf{u}, \quad-\nabla_{\ddagger} \cdot \mathbf{u}=g+h \beta^{-1} \mathbf{c}^{\prime \prime} \cdot \mathbf{u} \quad \text { in } \omega(s), \\
\mathbf{u}=\mathbf{h} \quad \text { on } \partial \omega(s) .
\end{gathered}
$$

Using the boundedness of $\beta^{-1}$ and (13), we have the following estimate due to results in [17].

$$
\begin{aligned}
\left\|\nabla_{\ddagger} \nabla_{\ddagger} \mathbf{u}\right\|_{\mathbf{L}^{2}(\omega(s))}+ & \left\|\nabla_{\ddagger} q\right\|_{\mathbf{L}^{2}(\omega(s))} \leq c\left(\|\mathbf{f}\|_{\mathbf{L}^{2}(\omega(s))}+h^{1 / 2} \lambda^{1 / 2}\left\|\nabla_{\ddagger} \mathbf{u}\right\|_{\mathbf{L}^{2}(\omega(s))}\right. \\
& \left.+\|g\|_{H^{1}(\omega(s))}+h^{1 / 2} \lambda^{1 / 2}\|\mathbf{u}\|_{\mathbf{H}^{1}(\omega(s))}+\|\mathbf{h}\|_{\mathbf{H}^{3 / 2}(\partial \omega(s))}\right) .
\end{aligned}
$$

Then applying (22), we get (23) by using (16).

We have the following corollary as a consequence of the above theorem.

Corollary 1. Given $\mathbf{f} \in \mathcal{C}^{1}\left((0,1), \mathbf{L}^{2}(\omega(s))\right), g \in \mathcal{C}^{1}\left((0,1), H^{1}(\omega(s))\right)$ and $\mathbf{h} \in \mathcal{C}^{1}\left((0,1), \mathbf{H}^{3 / 2}(\partial \omega(s))\right)$ such that (20) holds for every $s \in(0,1)$, then the solution of (21) satisfies the estimate

$$
\begin{gathered}
\left\|\left(\partial_{s} \mathbf{u}\right)_{\ddagger}\right\|_{\mathbf{H}^{1}(\omega(s))}+\left\|\partial_{s}(q-\bar{q})\right\|_{L^{2}(\omega(s))} \leq c\left(\left\|\left(\partial_{s} \mathbf{f}\right)_{\ddagger}\right\|_{\mathbf{H}^{-1}(\omega(s))}+\left\|\partial_{s} g\right\|_{L^{2}(\omega(s))}\right. \\
\quad+\left\|\partial_{s} \mathbf{h}\right\|_{\mathbf{H}^{1 / 2}(\partial \omega(s))}+(\lambda+\gamma)\left(\|\mathbf{f}\|_{\mathbf{L}^{2}(\omega(s))}+\|g\|_{H^{1}(\omega(s))}+\|\mathbf{h}\|_{\mathbf{H}^{3 / 2}(\partial \omega(s))}\right) .
\end{gathered}
$$

Proof. Differentiating (21) with respect to $s$, we get the system of equations

$$
\begin{gathered}
-\beta^{-1} \nabla_{\ddagger} \cdot \beta \nabla_{\ddagger}\left(\partial_{s} \mathbf{u}\right)_{\ddagger}+\nabla_{\ddagger}\left(\partial_{s} q\right)=\left(\partial_{s} \mathbf{f}\right)_{\ddagger}+\beta^{-1} \nabla_{\ddagger} \cdot\left(\partial_{s} \beta\right) \nabla_{\ddagger} \mathbf{u}-\beta^{-2}\left(\partial_{s} \beta\right) \nabla_{\ddagger} \cdot \beta \nabla_{\ddagger} \mathbf{u}, \\
-\beta^{-1} \nabla_{\ddagger} \cdot \beta\left(\partial_{s} \mathbf{u}\right)_{\ddagger}=\partial_{s} g+\beta^{-1} \nabla_{\ddagger} \cdot\left(\partial_{s} \beta\right) \mathbf{u}-\beta^{-2}\left(\partial_{s} \beta\right) \nabla_{\ddagger} \cdot \beta \mathbf{u} \quad \text { in } \omega(s), \\
\partial_{s} \mathbf{u}=\partial_{s} \mathbf{h}-\left(\partial_{s} R\right) \partial_{\eta} \mathbf{u} \quad \text { on } \quad \partial \omega(s) .
\end{gathered}
$$

If the condition (20) corresponding to the above system is satisfied, then we can apply Theorem 1.

Claim 1. For every $s \in(0,1)$,

$$
\begin{array}{r}
\int_{\omega(s)} \beta\left(\partial_{s} g+\beta^{-1} \nabla_{\ddagger} \cdot\left(\partial_{s} \beta\right) \mathbf{u}-\beta^{-2}\left(\partial_{s} \beta\right) \nabla_{\ddagger} \cdot \beta \mathbf{u}\right) \mathrm{d} \sigma(\eta, \theta) \\
+\int_{0}^{2 \pi} \beta\left(\partial_{s} \mathbf{h}-\left(\partial_{s} R\right) \partial_{\eta} \mathbf{u}\right) \cdot\left(R \mathbf{e}_{1}-\left(\partial_{\theta} R\right) \mathbf{e}_{2}\right) \mathrm{d} \theta=0 .
\end{array}
$$

The proof is presented in the appendix. Applying Theorem 1, we find

$$
\begin{aligned}
& \left\|\left(\partial_{s} \mathbf{u}\right)_{\ddagger}\right\|_{\mathbf{H}^{1}(\omega(s))}+\left\|\partial_{s}(q-\bar{q})\right\|_{L^{2}(\omega(s))} \leq c\left(\left\|\left(\partial_{s} \mathbf{f}\right)_{\ddagger}\right\|_{\mathbf{H}^{-1}(\omega(s))}\right. \\
& \left.\quad+\left\|\partial_{s} g\right\|_{L^{2}(\omega(s))}+\left\|\partial_{s} \mathbf{h}\right\|_{\mathbf{H}^{1 / 2}(\partial \omega(s))}+\lambda\left\|\nabla_{\ddagger} \mathbf{u}\right\|_{\mathbf{L}^{2}(\omega(s))}+\gamma\left\|\partial_{\eta} \mathbf{u}\right\|_{\mathbf{H}^{1 / 2}(\partial \omega(s))}\right)
\end{aligned}
$$

where we have used (9) and (11). Then we estimate $\left\|\nabla_{\ddagger} \mathbf{u}\right\|_{\mathbf{L}^{2}(\omega(s))}$ using (22) and note that $\left\|\partial_{\eta} \mathbf{u}\right\|_{\mathbf{H}^{1 / 2}(\partial \omega(s))} \leq\left\|\nabla_{\ddagger} \mathbf{u}\right\|_{\mathbf{H}^{1 / 2}(\partial \omega(s))}$. The right hand side in the preceding inequality is in fact a norm of the trace of the function $\nabla_{\ddagger} \mathbf{u}$, hence using the Trace theorem we have

$$
\left\|\nabla_{\ddagger} \mathbf{u}\right\|_{\mathbf{H}^{1 / 2}(\partial \omega(s))} \leq c\left\|\nabla_{\ddagger} \mathbf{u}\right\|_{\mathbf{H}^{1}(\omega(s))} .
$$

Subsequently, using (23), we get an upper bound on $\left\|\nabla_{\ddagger} \mathbf{u}\right\|_{\mathbf{H}^{1}(\omega(s))}$ which leads to (24).

\subsection{The Elliptic System}

The next theorem provides us the estimates for the model problem for scalar functions that appear in the asymptotic procedure. The results are standard (see e.g. [8]) and hence the proof is omitted. 
Theorem 2. Let there be given $f \in H^{-1}(\omega(s))$ and $k \in H^{1 / 2}(\partial \omega(s))$. Then there exists a unique $u \in$ $H^{1}(\omega(s))$ solving

$$
\begin{gathered}
-\beta^{-1} \nabla_{\ddagger} \cdot \beta \nabla_{\ddagger} u=f \quad \text { in } \quad \omega(s), \\
u=k \quad \text { on } \partial \omega(s) .
\end{gathered}
$$

The solution admits the following estimate:

$$
\|u\|_{H^{1}(\omega(s))} \leq c\left(\|f\|_{H^{-1}(\omega(s))}+\|k\|_{H^{1 / 2}(\partial \omega(s))}\right) .
$$

For general $n \geq 1$, if $f \in H^{n-2}(\omega(s))$ and $k \in H^{n-1 / 2}(\partial \omega(s))$, then $u \in H^{n}(\omega(s))$ satisfies

$$
\|u\|_{H^{n}(\omega(s))} \leq c\left(\|f\|_{H^{n-2}(\omega(s))}+\|k\|_{H^{n-1 / 2}(\partial \omega(s))}\right) .
$$

Consequently, we have the following corollary.

Corollary 2. Given $f \in \mathcal{C}^{2}\left((0,1), H^{n}(\omega(s))\right), k \in \mathcal{C}^{2}\left((0,1), H^{n+3 / 2}(\partial \omega(s))\right)$, the solution of (25) satisfies the estimates

$$
\begin{aligned}
& \left\|\partial_{s} u\right\|_{H^{n}(\omega(s))} \leq c\left(\left\|\partial_{s} f\right\|_{H^{n-2}(\omega(s))}+\left\|\partial_{s} k\right\|_{H^{n-1 / 2}(\partial \omega(s))}\right. \\
& \left.\quad+(\lambda+\gamma)\left(\|f\|_{H^{n-1}(\omega(s))}+\|k\|_{H^{n+1 / 2}(\partial \omega(s))}\right)\right)
\end{aligned}
$$

and

$$
\begin{aligned}
& \left\|\partial_{s}^{2} u\right\|_{H^{n}(\omega(s))} \leq c\left(\left\|\partial_{s}^{2} f\right\|_{H^{n-2}(\omega(s))}+\left\|\partial_{s}^{2} k\right\|_{H^{n-1 / 2}(\partial \omega(s))}\right. \\
& \quad+(\lambda+\gamma)\left(\left\|\partial_{s} f\right\|_{H^{n-1}(\omega(s))}+\left\|\partial_{s} k\right\|_{H^{n+1 / 2}(\partial \omega(s))}\right) \\
& \left.\quad+\left(\lambda^{*}+\gamma^{*}+h^{-1 / 2} \lambda^{3 / 2}+\gamma^{2}\right)\left(\|f\|_{H^{n}(\omega(s))}+\|k\|_{H^{n+3 / 2}(\partial \omega(s))}\right)\right) .
\end{aligned}
$$

Proof. To prove (28), we follow identical steps as in the proof of Corollary 1. To prove (29), we differentiate (25) twice with respect to $s$ to obtain

$$
\begin{aligned}
& -\beta^{-1} \nabla_{\ddagger} \cdot \beta \nabla_{\ddagger} \partial_{s}^{2} u=\partial_{s}^{2} f+2 \beta^{-1} \nabla_{\ddagger} \cdot\left(\partial_{s} \beta\right) \nabla_{\ddagger} \partial_{s} u-2 \beta^{-2}\left(\partial_{s} \beta\right) \nabla_{\ddagger} \cdot \beta \nabla_{\ddagger} \partial_{s} u \\
& -2 \beta^{-2}\left(\partial_{s} \beta\right) \nabla_{\ddagger} \cdot\left(\partial_{s} \beta\right) \nabla_{\ddagger} u+\beta^{-1} \nabla_{\ddagger} \cdot\left(\partial_{s}^{2} \beta\right) \nabla_{\ddagger} u-\left(\partial_{s}\left(\beta^{-2} \partial_{s} \beta\right)\right) \nabla_{\ddagger} \cdot \beta \nabla_{\ddagger} u \quad \text { in } \quad \omega(s), \\
& \partial_{s}^{2} u=\partial_{s}^{2} k-2\left(\partial_{s} R\right) \partial_{\eta} \partial_{s} u-\left(\partial_{s}^{2} R\right) \partial_{\eta} u-\left(\partial_{s} R\right)^{2} \partial_{\eta}^{2} \mathbf{u} \quad \text { on } \quad \partial \omega(s) .
\end{aligned}
$$

Then we apply Theorem 2 and use (11), (15), (9) and (10) to get

$$
\begin{aligned}
& \left\|\partial_{s}^{2} u\right\|_{H^{1}(\omega(s))} \leq c\left(\left\|\partial_{s}^{2} f\right\|_{H^{-1}(\omega(s))}+\left(\lambda^{2}+\lambda^{*}+h^{-1 / 2} \lambda^{3 / 2}\right)\left\|\nabla_{\ddagger} u\right\|_{L^{2}(\omega(s))}\right. \\
& \quad+\lambda\left\|\nabla_{\ddagger} \partial_{s} u\right\|_{L^{2}(\omega(s))}+\left\|\partial_{s}^{2} k\right\|_{H^{1 / 2}(\partial \omega(s))}+\gamma\left\|\partial_{\eta} \partial_{s} u\right\|_{H^{1 / 2}(\partial \omega(s))} \\
& \left.\quad+\gamma^{*}\left\|\partial_{\eta} u\right\|_{H^{1 / 2}(\partial \omega(s))}+\gamma^{2}\left\|\partial_{\eta}^{2} u\right\|_{H^{1 / 2}(\partial \omega(s))}\right) .
\end{aligned}
$$

Estimating the right hand side with the help of (26), (27) and (28) and using (16), we arrive at (29).

\subsection{The Divergence Equation}

In this subsection, we consider the divergence equation for two different cases of a curvilinear pipe having a variable cross-section. The divergence equation frequently appears in the study of flows and hence is an important auxiliary problem, see $[4,7]$. For the case of thin tubular domains, in the previous works starting with [11], coordinate dilation and uniform scaling of the transversal velocity components were sufficient to derive the specific estimates. See also [15]. The presence of curvature complicates our case, therefore, position dependent scaling involving the curvature dependent scale factor $\beta$ is introduced to tackle this problem.

Firstly, we present a Lemma about the divergence equation in a thin curvilinear pipe $\Omega^{h}$ laving length 1. 
Lemma 1. Let there be $f \in L^{2}\left(\Omega^{h}\right)$ such that

$$
\int_{\Omega^{h}} f \mathrm{~d} \mathbf{x}=0 .
$$

Then there exists a (non unique) solution $\mathbf{w} \in \mathbf{H}^{1}\left(\Omega^{h}\right)$ of the divergence equation

$$
\begin{aligned}
-\nabla_{\mathbf{x}} \cdot \mathbf{w}=f & \text { in } \Omega^{h}, \\
\mathbf{w}=\mathbf{0} & \text { on } \partial \Omega^{h},
\end{aligned}
$$

which obeys the estimate

$$
\begin{gathered}
\left\|\nabla \cdot \mathbf{w}_{\ddagger}\right\|_{L^{2}\left(\Omega^{h}\right)}+h^{-1}\left\|\mathbf{w}_{\ddagger}\right\|_{L^{2}\left(\Omega^{h}\right)}+h\left\|\nabla \cdot w_{3}\right\|_{L^{2}\left(\Omega^{h}\right)} \\
+\left\|\partial_{s} w_{3}\right\|_{L^{2}\left(\Omega^{h}\right)}+\left\|w_{3}\right\|_{L^{2}\left(\Omega^{h}\right)} \leq C\|f\|_{L^{2}\left(\Omega^{h}\right)}, \\
h^{-1}\left\|\left(\partial_{s} \mathbf{w}_{\ddagger}\right)_{\ddagger}\right\|_{L^{2}\left(\Omega^{h}\right)} \leq C(1+\lambda)\|f\|_{L^{2}\left(\Omega^{h}\right)}
\end{gathered}
$$

for some constant $C$ independent of $f$ and $h$.

Proof. Noting the fact that $\nabla_{\ddagger} \beta=-h \mathbf{c}^{\prime \prime}$ and in accordance with the scaled parameter $\eta=h^{-1} r$, we introduce the scaled function

$$
\hat{\mathbf{w}}=h^{-1} \beta \mathbf{w}_{\ddagger}+w_{3} \mathbf{c}^{\prime} .
$$

Thus, we have

$$
\begin{aligned}
\nabla_{\mathbf{x}} \cdot \mathbf{w} & =h^{-1} \nabla_{\ddagger} \cdot \mathbf{w}_{\ddagger}+\beta^{-1}\left(\partial_{s} w_{3}-\mathbf{c}^{\prime \prime} \cdot \mathbf{w}_{\ddagger}\right)=\beta^{-1}\left(\nabla_{\ddagger} \cdot \hat{\mathbf{w}}_{\ddagger}+\partial_{s} \hat{w}_{3}\right) \\
& =\beta^{-1}\left(\partial_{\eta} \hat{w}_{1}+\eta^{-1} \hat{w}_{1}+\eta^{-1} \partial_{\theta} \hat{w}_{2}+\partial_{s} \hat{w}_{3}\right) .
\end{aligned}
$$

Clearly, the terms within the brackets in the last equality represent the polar form of the divergence of a vector field defined in a straight cylinder. As a result, we can say that $\mathbf{w}$ satisfies (31) if and only if $\overline{\mathbf{w}}:=\hat{w}_{1} \hat{\boldsymbol{\eta}}+\hat{w}_{2} \hat{\boldsymbol{\theta}}+\hat{w}_{3} \hat{\mathbf{s}}$ (likewise for $\hat{\boldsymbol{\eta}}, \hat{\boldsymbol{\theta}}$ and $\hat{\mathbf{s}}$ being the unit vectors corresponding to cylindrical coordinates) satisfies the system

$$
\begin{aligned}
\operatorname{div} \overline{\mathbf{w}} & =\beta f \quad \text { in } \Xi, \\
\overline{\mathbf{w}} & =\mathbf{0} .
\end{aligned}
$$

Here $\Xi$ is a cylinder with a straight axis and given as

$$
\Xi:=\left\{\mathbf{x}(\eta, \theta, s)=(\eta \cos \theta, \eta \sin \theta, s): 0 \leq \eta \leq h^{-1} R(\theta, s), 0 \leq \theta<2 \pi, 0<s<1\right\} .
$$

For the function $\beta f$, we have that

$$
\int_{\Xi} \beta f \mathrm{~d} \mathbf{x}=\int_{0}^{1} \int_{0}^{2 \pi} \int_{0}^{h^{-1}} \beta f \eta \mathrm{d} \eta \mathrm{d} \theta \mathrm{d} s=\int_{\Omega^{h}} f \mathrm{~d} \mathbf{x}=0 .
$$

Thus the compatibility condition is met by $\beta f$.

Therefore, by a classical result on the divergence equation (see [6]) in a fixed Lipschitz domain, we have $\overline{\mathbf{w}} \in \mathbf{H}^{1}(\Xi) \Rightarrow \hat{\mathbf{w}} \in \mathbf{H}^{1}\left(\Omega^{h}\right)$ and for a constant $C$ independent of the data, the estimate

$$
\begin{aligned}
& \left\|\nabla_{\ddagger} \hat{\mathbf{w}}_{\ddagger}\right\|_{\mathbf{L}^{2}\left(\Omega^{h}\right)}+\left\|\left(\partial_{s} \hat{\mathbf{w}}_{\ddagger}\right)\right\|_{\ddagger}\left\|_{\mathbf{L}^{2}\left(\Omega^{h}\right)}+\right\| \hat{\mathbf{w}}_{\ddagger} \|_{\mathbf{L}^{2}\left(\Omega^{h}\right)} \\
& \quad+\left\|\nabla_{\ddagger} \hat{w}_{3}(f)\right\|_{\mathbf{L}^{2}\left(\Omega^{h}\right)}+\left\|\partial_{s} \hat{w}_{3}(f)\right\|_{L^{2}\left(\Omega^{h}\right)}+\left\|\hat{w}_{3}(f)\right\|_{L^{2}\left(\Omega^{h}\right)} \leq C\|f\|_{L^{2}\left(\Omega^{h}\right)} .
\end{aligned}
$$

Owing to the bounds (11) and (13), the above leads us to (1).

We present another lemma on the divergence equation restricted to a length of a pipe that is comparable to the thickness of the pipe. The estimate in this case is modified as compared to that in Lemma 1 due to the differing aspect ratio of the segment of the curvilinear pipe in question. Let us consider (31) and (30) restricted to the domain $\Omega_{\text {end }}^{h}:=\left\{\mathbf{x}(r, \theta, s) \in \Omega^{h}: 0<s<l, l=O(h)\right\}$. 
Lemma 2. Let $f \in L^{2}\left(\Omega_{\text {end }}^{h}\right)$ satisfy

$$
\int_{\Omega_{\text {end }}^{h}} f \mathrm{~d} \mathbf{x}=0 .
$$

Then there exists a (non unique) solution $\mathbf{w} \in \mathbf{H}^{1}\left(\Omega_{\text {end }}^{h}\right)$ of the divergence equation

$$
\begin{aligned}
-\nabla_{\mathbf{x}} \cdot \mathbf{w} & =f \text { in } \Omega_{e n d}^{h}, \\
\mathbf{w} & =\mathbf{0} \text { on } \partial \Omega_{e n d}^{h},
\end{aligned}
$$

which obeys the estimate

$$
\begin{aligned}
& \left\|\nabla \cdot \mathbf{w}_{\ddagger}\right\|_{\mathbf{L}^{2}\left(\Omega_{e n d}^{h}\right)}+\left\|\left(\partial_{s} \mathbf{w}_{\ddagger}\right)_{\ddagger}\right\|_{\mathbf{L}^{2}\left(\Omega_{e n d}^{h}\right)}+h^{-1}\left\|\mathbf{w}_{\ddagger}\right\|_{\mathbf{L}^{2}\left(\Omega_{e n d}^{h}\right)} \\
& \quad+\left\|\nabla \cdot w_{3}\right\|_{\mathbf{L}^{2}\left(\Omega_{e n d}^{h}\right)}+\left\|\partial_{s} w_{3}\right\|_{L^{2}\left(\Omega_{e n d}^{h}\right)}+h^{-1}\left\|w_{3}\right\|_{L^{2}\left(\Omega_{e n d}^{h}\right)} \leq C\|f\|_{L^{2}\left(\Omega_{e n d}^{h}\right)},
\end{aligned}
$$

for some constant $C$ independent of $f$ and $h$.

Proof. Along with the scaled radial parameter $\eta=h^{-1} r$, we consider the scaled longitudinal parameter $\tau=h^{-1} s$ and the scaled function

$$
\hat{\mathbf{w}}=\beta \mathbf{w}_{\ddagger}+w_{3} \mathbf{c}^{\prime} .
$$

The rest of the proof follows the steps in the proof of Lemma 1 and we get the required estimate.

\section{Formal Asymptotic Procedure}

Let us consider the asymptotic Ansätze:

$$
\begin{aligned}
& p^{h}(r, \theta, s)=h^{-3} p^{0}(s)+h^{-2} p^{1}(\eta, \theta, s)+h^{-1} p^{2}(\eta, \theta, s)+\cdots \\
& \mathbf{v}^{h}(r, \theta, s)=h^{-1} \mathbf{v}^{1}(\eta, \theta, s)+h^{0} \mathbf{v}^{2}(\eta, \theta, s)+\cdots
\end{aligned}
$$

Having an $O\left(h^{-1}\right)$ velocity still results in an $O(h)$ flux through the cross-sections so that ignoring the convective term in the Navier-Stokes equations can still be justified.

The first step of matching coefficients of the leading order of $h$ in (17), (19) and (3) produces the following system of equations:

$$
\begin{aligned}
& -\beta^{-1} \nabla_{\ddagger} \cdot \beta \nabla_{\ddagger} \mathbf{v}_{\ddagger}^{1}+\nabla_{\ddagger} p^{1}=\mathbf{0}_{\ddagger}, \quad-\beta^{-1} \nabla_{\ddagger} \cdot \beta \mathbf{v}_{\ddagger}^{1}=0 \quad \text { in } \quad \omega(s), \\
& \mathbf{v}_{\ddagger}^{1}=\mathbf{0}_{\ddagger} \quad \text { on } \quad \partial \omega(s) .
\end{aligned}
$$

The solution is of the form $\mathbf{v}_{\ddagger}^{1}=\mathbf{0}_{\ddagger}$ and $p^{1}=p^{1}(s)$.

For the third component, due to (18) and (3), we have the equations

$$
\begin{aligned}
& -\beta^{-1} \nabla_{\ddagger} \cdot \beta \nabla_{\ddagger} v_{3}^{1}+\beta^{-1} \partial_{s} p^{0}=0 \quad \text { in } \omega(s), \\
& v_{3}^{1}=0 \quad \text { on } \quad \partial \omega(s) .
\end{aligned}
$$

Its solution takes the form

$$
v_{3}^{1}=-\frac{1}{2} \Psi(\eta, \theta, s) \partial_{s} p^{0}(s),
$$

where $\Psi$ is a function (Prandtl function in case of $\beta \equiv 1$ ) satisfying

$$
-\beta^{-1} \nabla_{\ddagger} \cdot \beta \nabla_{\ddagger} \Psi=2 \beta^{-1} \quad \text { in } \quad \omega(s), \quad \Psi=0 \quad \text { on } \quad \partial \omega(s) .
$$

For the solution of (39), due to (26), we have the estimate

$$
\|\Psi\|_{H^{1}(\omega(s))} \leq c\left\|\beta^{-1}\right\|_{H^{-1}(\omega(s))} \leq c .
$$


Applying Corollary 2 and using (13) and (15), we obtain the additional estimates

$$
\begin{aligned}
\left\|\partial_{s} \Psi\right\|_{L^{2}(\omega(s))} \leq & c\left[\left\|\partial_{s}\left(\beta^{-1}\right)\right\|_{H^{-1}(\omega(s))}+(\lambda+\gamma)\left\|\beta^{-1}\right\|_{L^{2}(\omega(s))}\right] \leq c(\lambda+\gamma) . \\
\left\|\partial_{s}^{2} \Psi\right\|_{L^{2}(\omega(s)) \leq} \leq & c\left[\left\|\partial_{s}^{2}\left(\beta^{-1}\right)\right\|_{H^{-1}(\omega(s))}+(\lambda+\gamma)\left\|\partial_{s}\left(\beta^{-1}\right)\right\|_{L^{2}(\omega(s))}\right. \\
& \left.+\left(\lambda^{*}+\gamma^{*}+h^{-1 / 2} \lambda^{3 / 2}+\gamma^{2}\right)\left\|\beta^{-1}\right\|_{H^{1}(\omega(s))}\right] \\
\leq & c\left(\lambda^{*}+\gamma^{*}+h^{-1 / 2} \lambda^{3 / 2}+\gamma^{2}\right) .
\end{aligned}
$$

We also need the boundedness of the functions $\Psi$ and $\Psi^{-1}$ to proceed further and hence we present the following proposition.

Proposition 1. There exist constants $\mathfrak{C}_{1}, \mathfrak{C}_{2}>0$ dependent on the domain $\omega$ such that

$$
\mathfrak{C}_{1} \leq \Psi \leq \mathfrak{C}_{2}
$$

Proof. For a bounded domain $\omega$, let us consider a general elliptic operator

$$
L=-\sum_{i, j=1}^{2} \partial_{x_{i}}\left(a^{i j} \partial_{x_{i}}\right)
$$

The coefficients $a^{i j}$ are real-valued from $L^{\infty}(\omega)$ and satisfy

$$
\mu_{1}|\xi|^{2} \leq \sum_{i, j=1}^{2} a^{i j} \xi_{i}^{*} \xi_{j} \leq \mu_{2}|\xi|^{2}
$$

for some $\mu_{1}, \mu_{2}>0$. Let $\mathcal{G}=\mathcal{G}(x, y)$ be the Green's function for $L$ with the homogeneous boundary condition on $\partial \omega$. Then, $\mathcal{G}>0$ and for $x, y \in \omega$,

$$
\mathcal{G}(x, y) \leq C_{1}(|\ln | x-y||+1)
$$

and

$$
\mathcal{G}(x, y) \geq C_{2}(|\ln | x-y||+1)
$$

for $|x-y| \leq \frac{1}{2} \operatorname{dist}(y, \partial \omega)$. By results in [9], it is sufficient to verify this for the Laplacian for which it is known. Here, $C_{1}$ and $C_{2}$ are positive constants that depend only on $\mu_{1}$ and $\mu_{2}$. The function $\Psi$ is represented as

$$
\Psi(x)=2 \int_{\omega} \mathcal{G}(x, y) \mathrm{d} y,
$$

where $\mathcal{G}$ now represents the Green's function for the operator $\beta^{-1} \nabla_{\ddagger} \cdot \beta \nabla_{\ddagger}$. The required estimate follows from (43) and (44).

We define the generalized torsional rigidity

$$
G(s):=2 \int_{\omega(s)} \Psi(\eta, \theta, s) \mathrm{d} \sigma(\eta, \theta)=\int_{\omega(s)} \beta(\eta, \theta, s)\left|\nabla_{\ddagger} \Psi(\eta, \theta, s)\right|^{2} \mathrm{~d} \sigma(\eta, \theta)>0 .
$$

Due to this definition and the boundedness of the domain $\omega(s)$, Proposition 1 guarantees the existence of constants $A, B$ such that $0<A \leq G(s) \leq B<\infty$ for all $s \in[0,1]$.

Now we consider the next step in the asymptotic procedure, that is to compare the coefficients of the next order (with respect to $h$ ) terms. We have

$$
\begin{aligned}
& -\beta^{-1} \nabla_{\ddagger} \cdot \beta \nabla_{\ddagger} \mathbf{v}_{\ddagger}^{2}+\nabla_{\ddagger} p^{2}=\beta^{-2} h \mathbf{c}_{\ddagger}^{\prime \prime \prime} v_{3}^{1}+\beta^{-3} h \mathbf{c}^{\prime \prime}\left(2 \beta \partial_{s} v_{3}^{1}-v_{3}^{1} \partial_{s} \beta\right), \\
& -\nabla_{\ddagger} \cdot \beta \mathbf{v}_{\ddagger}^{2}=\partial_{s} v_{3}^{1} \quad \text { in } \quad \omega(s), \quad \mathbf{v}_{\ddagger}^{2}=\mathbf{0}_{\ddagger} \quad \text { on } \quad \partial \omega(s) .
\end{aligned}
$$


Owing to the zero boundary conditions for $\mathbf{v}_{\ddagger}^{2}$ and $\Psi$, we get the compatibility condition for this problem

$$
\begin{aligned}
0 & =\int_{\omega(s)} \nabla_{\ddagger} \cdot \beta \mathbf{v}_{\ddagger}^{2} \mathrm{~d} \sigma(\eta, \theta)=-\int_{\omega(s)} \partial_{s} v_{3}^{1} \mathrm{~d} \sigma(\eta, \theta) \\
& =\frac{1}{2} \int_{\omega(s)} \partial_{s}\left(\Psi(\eta, \theta, s) \partial_{s} p^{0}(s)\right) \mathrm{d} \sigma(\eta, \theta)=\frac{1}{2} \partial_{s}\left(\int_{\omega(s)} \Psi(\eta, \theta, s) \mathrm{d} \sigma(\eta, \theta) \partial_{s} p^{0}(s)\right) .
\end{aligned}
$$

Thus, we have derived the modified Reynolds equation

$$
-\partial_{s}\left(G(s) \partial_{s} p^{0}(s)\right)=0, \quad s \in(0,1) .
$$

Remark 2. In the absence of curvature, i.e. $\beta \equiv 1,(47)$ is the classical Reynolds equation, cf. [12].

This motivates the imposition of the boundary flux condition

$$
\begin{gathered}
\int_{\omega(0)} v_{3}^{h}(r, \theta, 0) \mathrm{d} \sigma(\eta, \theta)=h^{-1} F^{0} \\
\Rightarrow-G(0) \partial_{s} p^{0}(0)=4 F^{0},
\end{gathered}
$$

where $F^{0}$ denotes the prescribed total volumetric flow rate through the inlet of the unscaled domain $\Omega^{h}$. We can also argue to impose the condition

$$
p^{0}(1)=p_{\text {per }}^{0} .
$$

The mixed boundary problem stated in (47), (48) and (49) has the solution

$$
p^{0}(s)=p_{p e r}^{0}+4 F^{0} \int_{s}^{1} G(t)^{-1} \mathrm{~d} t .
$$

This leads to

$$
\left|\partial_{s} p^{0}(s)\right|=\left|4 F^{0} G(s)^{-1}\right| \leq c
$$

as well as

$$
\left|\partial_{s}^{2} p^{0}(s)\right|=\left|4 F^{0} G(s)^{-2} \partial_{s} G(s)\right| \leq c\left\|\partial_{s} \Psi\right\|_{L^{2}(\omega(s))} \leq c(\lambda+\gamma)
$$

where we used (41). Similarly,

$$
\left|\partial_{s}^{3} p^{0}(s)\right| \leq c\left(\left\|\left(\partial_{s} R\right) \partial_{s} \Psi\right\|_{L^{2}(\partial \omega(s))}+\left\|\partial_{s}^{2} \Psi\right\|_{L^{2}(\omega(s))} \leq c\left(\lambda^{*}+\gamma^{*}+h^{-1 / 2} \lambda^{3 / 2}+\gamma^{2}\right)\right.
$$

where we have used (42) and the fact that $\left\|\partial_{s} \Psi\right\|_{L^{2}(\partial \omega(s))} \leq c\left\|\partial_{s} \Psi\right\|_{H^{1}(\partial \omega(s))}$.

As a result of the above along with (40), (41) and (42), (38) gives us

$$
\begin{aligned}
& \left\|v_{3}^{1}\right\| \leq \operatorname{ch}, \quad\left\|\partial_{s} v_{3}^{1}\right\| \leq \operatorname{ch}(\lambda+\gamma) \\
& \quad \text { and } \quad\left\|\partial_{s}^{2} v_{3}^{1}\right\| \leq \operatorname{ch}\left(\lambda^{*}+\gamma^{*}+h^{-1 / 2} \lambda^{3 / 2}+\gamma^{2}\right) .
\end{aligned}
$$

Here and henceforth, $\|\cdot\|$ denotes the usual norm in $L^{2}\left(\Omega^{h}\right)$ and all constants $c$ will be of the form $c=C\left(\left|F^{0}\right|+\left|p_{\text {per }}^{0}\right|\right)$ where $C$ is independent of $F^{0}, p_{\text {per }}^{0}$.

Consequently, by (22), since $\partial_{s} v_{3}^{1} \in L^{2}(\omega(s))$ and $v_{3}^{1} \in L^{2}(\omega(s)) \subset H^{-1}(\omega(s))$, denoting the average of $p^{2}$ over the cross-section by $\bar{p}^{2}$ we have for the solution of (46),

$$
\begin{gathered}
\left\|\mathbf{v}_{\ddagger}^{2}\right\|_{H^{1}(\omega(s))}+\left\|p^{2}-\bar{p}^{2}\right\|_{L^{2}(\omega(s))} \leq c\left(\lambda\left\|v_{3}^{1}\right\|_{H^{-1}(\omega(s))}+\left\|\partial_{s} v_{3}^{1}\right\|_{L^{2}(\omega(s))}\right) \\
\Rightarrow\left\|\nabla_{\ddagger} \mathbf{v}_{\ddagger}^{2}\right\|+\left\|\mathbf{v}_{\ddagger}^{2}\right\|+\left\|p^{2}-\bar{p}^{2}\right\| \leq \operatorname{ch}(\lambda+\gamma)
\end{gathered}
$$


and similarly by (24)

$$
\begin{gathered}
\left\|\left(\partial_{s} \mathbf{v}_{\ddagger}^{2}\right)_{\ddagger}\right\|_{H^{1}(\omega(s))}+\left\|\partial_{s}\left(p^{2}-\bar{p}^{2}\right)\right\|_{L^{2}(\omega(s))} \leq \operatorname{ch}\left(\lambda^{*}+\gamma^{*}+h^{-1 / 2} \lambda^{3 / 2}+\gamma^{2}\right) \\
\Rightarrow\left\|\partial_{s} \mathbf{v}_{\ddagger}^{2}\right\|=\left\|\left(\partial_{s} \mathbf{v}_{\ddagger}^{2}\right)_{\ddagger}-\mathbf{c}^{\prime \prime} \cdot \mathbf{v}_{\ddagger}^{2} \mathbf{c}^{\prime}\right\| \leq \operatorname{ch}\left(\lambda^{*}+\gamma^{*}+h^{-1} \lambda+\gamma^{2}\right) .
\end{gathered}
$$

\section{Boundary Conditions at the Ends}

In order to solve the Stokes problem, we need to specify appropriate boundary conditions at the inlet and the outlet. We consider the domain $\Omega^{h}$ to be an arbitrarily chosen segment of a much larger pipe in which the fluid is injected at one end and it flows out at the other. Such conditions at the end cross-sections are extremely difficult to model reasonably hence we restrict ourselves to the chosen segment, possibly far away from the ends. Imposing artificial boundary conditions at the ends of the chosen segment gives rise to the boundary layer phenomena near those ends. It brings about a quick variability near the end cross-sections in the solution $\left\{\mathbf{v}^{h}, p^{h}\right\}$ of the problem. Although, from a practical point of view, it is absurd to expect such quick variability at arbitrarily chosen portions of the full pipe.

The function of the boundary layer terms in the solutions is to reduce the discrepancy in the artificial boundary conditions. We want to impose such boundary conditions which make the discrepancy as small as possible. The boundary conditions chosen must as well be included in the Green formula for the Stokes operator. One can of course formulate elaborate sets of conditions to achieve this. We however, opt for the simpler way of preparing the boundary data in accordance to our approximations. We take the traces of our approximate fields at the end cross-sections and use them as the boundary data. Thus we reduce the discrepancy at the boundaries to zero while also diminishing the error estimates.

\subsection{Boundary Conditions on the Cross-Section $\omega^{h}(0)$}

We note that the components of the Ansätze (36) at the point $s=0$ are completely determined by the data of the problem. Indeed, according to the boundary condition (48) we have

$$
v_{3}^{1}(\eta, \theta, 0)=-\frac{1}{2} \Psi(\eta, \theta, 0) \partial_{s} p^{0}(0)=2 G(0)^{-1} F^{0} \Psi(\eta, \theta, 0) .
$$

Thus, we should take the boundary conditions

$$
\begin{aligned}
v_{3}^{h}(r, \theta, 0) & =2 h^{-1} G(0)^{-1} F^{0} \Psi(\eta, \theta, 0), \\
\mathbf{v}_{\ddagger}^{h}(r, \theta, 0) & =\mathbf{0}_{\ddagger} \quad \text { on } \quad \omega^{h}(0) .
\end{aligned}
$$

\subsection{Boundary Conditions on the Cross-Section $\omega^{h}(1)$}

Since the fluxes through the cross-sections do not change, the expressions (so called velocities of pseudodeformations) generated by the ansatz (36),

$$
h^{-1} \beta^{-1} \partial_{s} v_{3}^{1}(\eta, \theta, 1)-h^{-3} p^{0}(1), \quad h^{-1} \beta^{-1} \partial_{s} v_{j}^{2}(\eta, \theta, 1), \quad j=1,2,
$$

can be also evaluated by using the problem's data. The term $h^{-3} p^{0}(1)$ is essentially $\left(h^{-2}\right.$ times) larger than the other in (55). Therefore, we can take

$$
\beta^{-1} \partial_{s} v_{3}^{h}(r, \theta, 1)-p^{h}(r, \theta, 1)=-p_{p e r}^{h} \quad \text { on } \quad \omega^{h}(1)
$$

as one of the boundary conditions on the cross-section $\omega^{h}(1)$ with $p_{p e r}^{h}=h^{-3} p_{p e r}^{0}$. We emphasize that the pressure itself can be taken in the boundary condition since it does not appear in the Green formula for the Stokes system alone. Further, we complement (56) by the following conditions:

$$
v_{\ddagger}^{h}(r, \theta, 1)=\mathbf{0}_{\ddagger} \quad \text { on } \quad \omega^{h}(1) .
$$


Due to the continuity equation (2) and relation (57), we have

$$
0=\nabla_{\mathbf{x}} \cdot v^{h}(r, \theta, 1)=\beta^{-1} \partial_{s} v_{3}^{h}(r, \theta, 1)
$$

and hence the boundary conditions (56) and (57) lead to a constant pressure on the cross-section $\omega^{h}(1)$.

\section{Estimates of the Asymptotical Remainder Terms in the Stokes Problem}

Recall that the solution $\left(\mathbf{v}^{h}, p^{h}\right)$ of the problem (1)-(3) is represented as

$$
\mathbf{v}^{h}=\mathbf{v}^{h}+\mathbf{v}_{r e m}^{h} \quad \text { and } \quad p^{h}=p^{h}+p_{r e m}^{h}
$$

where we take the approximate solution to be

$$
\begin{aligned}
& \mathbb{p}^{h}(\mathbf{x})=h^{-3} p^{0}(s), \\
& \mathbf{v}^{h}(\mathbf{x})=h^{-1} v_{3}^{1}(\eta, \theta, s) \mathbf{c}^{\prime}(s)+X^{h}(s) \mathbf{v}_{\ddagger}^{2}(\eta, \theta, s),
\end{aligned}
$$

where $X^{h} \in C^{\infty}(0,1)$ is a cut-off function such that $0 \leq X^{h} \leq 1,\left|\partial_{s}^{p} X^{h}(s)\right| \leq c h^{-p}$,

$$
X^{h}(s)= \begin{cases}1 & \text { for } s \in(2 h, 1-2 h), \\ 0 & \text { for } s \in(0, h) \cup(1-h, 1)\end{cases}
$$

and $v_{3}^{1}$ and $\mathbf{v}_{\ddagger}^{2}$ are solutions of (37) and (46) respectively. Inclusion of the term $\mathbf{v}_{\ddagger}^{2}$ makes the approximate velocity divergence-free inside the channel away from the ends. Moreover, the order of magnitude of $\left|\mathbf{v}_{\ddagger}^{2}\right|$ grows closer to that of the leading term $h^{-1} v_{3}^{1}$ with increasing $\lambda$ as is evident from (50) and (51). Due to the restrictions $v_{3}^{1}=0, \mathbf{v}_{\ddagger}^{2}=0$ on $\partial \omega(z)$, the boundary condition (3) is met. Introducing the cut-off function ensures that the conditions (54) and (57) are fulfilled. The same is true for the condition (53) due to (38) and (48).

In order to derive estimates of the error terms, we require an approximate velocity that is divergencefree in the entire domain including near the ends. Hence, to compensate for the error in the divergence of $\mathbf{v}^{h}$ near the inlet and the outlet, we consider $\mathbf{w}$ which satisfies

$$
\begin{aligned}
-\nabla_{\mathbf{x}} \cdot \mathbf{w} & =h^{-1} \beta^{-1}\left(1-X^{h}\right) \partial_{s} v_{3}^{1} & & \text { in } \Omega^{h}, \\
\mathbf{w} & =\mathbf{0} & & \text { on } \partial \Omega^{h} .
\end{aligned}
$$

The compatibility condition for this problem is satisfied as

$$
\int_{\Omega^{h}} h^{-1} \beta^{-1}\left(1-X^{h}\right) \partial_{s} v_{3}^{1} \mathrm{~d} \mathbf{x}=\int_{0}^{1}\left(1-X^{h}\right) h \int_{\omega(s)} \partial_{s} v_{3}^{1} \mathrm{~d} \sigma(\eta, \theta) \mathrm{d} s=0 .
$$

Therefore one could apply Lemma 1 to get the corresponding estimates for the vector field $\mathbf{w}$. However, the estimates can be improved upon by observing that the right hand side vanishes in most of the domain. As $\operatorname{supp}\left(1-X^{h}\right) \subseteq[0,2 h] \cup[1-2 h, 1]$, it suffices to solve the above problem (58) in the region $\left\{\mathbf{x} \in \Omega^{h}: s \in(0,2 h) \cup(1-2 h, 1)\right\}$ with $\mathbf{w}$ vanishing on the boundary and then to extend it to the rest of $\Omega^{h}$ by setting $\mathbf{w}=\mathbf{0}$ for $s \in[2 h, 1-2 h]$. Note that the compatibility condition (33) is satisfied at the above mentioned region for the function $f=h^{-1} \beta^{-1}\left(1-X^{h}\right) \partial_{s} v_{3}^{1}$. Since this new domain where (58) needs to be solved, has comparable size in all directions (order $h$ ), we may use (35) to conclude

$$
\begin{aligned}
& \left\|\nabla \cdot \mathbf{w}_{\ddagger}\right\|+\left\|\left(\partial_{s} \mathbf{w}_{\ddagger}\right)_{\ddagger}\right\|+h^{-1}\left\|\mathbf{w}_{\ddagger}\right\|+\left\|\nabla \cdot w_{3}\right\|+\left\|\partial_{s} w_{3}\right\|+h^{-1}\left\|w_{3}\right\| \\
& \quad \leq c\left\|h^{-1} \beta^{-1}\left(1-X^{h}\right) \partial_{s} v_{3}^{1}\right\| \leq c h^{1 / 2}(\lambda+\gamma) .
\end{aligned}
$$

Let us denote the inner product in $L^{2}\left(\Omega^{h}\right)$ by $(\cdot, \cdot)$. The discrepancy in (1) is

$$
\begin{aligned}
\mathbf{F}^{h} & :=\Delta_{\mathbf{x}}\left(\mathbf{v}^{h}-\mathbf{v}^{h}\right)-\nabla_{\mathbf{x}}\left(p^{h}-\mathbb{p}^{h}\right)=-\Delta_{\mathbf{x}} \mathbf{v}^{h}+\nabla_{\mathbf{x}} \mathbb{p}^{h} \\
& =-\left(h^{-2} \beta^{-1} \nabla_{\ddagger} \cdot \beta \nabla_{\ddagger}+\beta^{-1} \partial_{s} \beta^{-1} \partial_{s}\right)\left(X^{h} \mathbf{v}_{\ddagger}^{2}+h^{-1} \mathbf{c}^{\prime} v_{3}^{1}\right)+\left(h^{-1} \nabla_{\ddagger}+\beta^{-1} \mathbf{c}^{\prime} \partial_{s}\right)\left(h^{-3} p^{0}\right) .
\end{aligned}
$$


Rearranging the terms with respect to orders of $h$, we have

$$
\begin{aligned}
\mathbf{F}^{h}= & -\beta^{-1} \partial_{s} \beta^{-1} \partial_{s}\left(X^{h} \mathbf{v}_{\ddagger}^{2}\right)-h^{-1} \beta^{-1} \partial_{s} \beta^{-1} \partial_{s}\left(\mathbf{c}^{\prime} v_{3}^{1}\right) \\
& -h^{-2} X^{h} \beta^{-1} \nabla_{\ddagger} \cdot \beta \nabla_{\ddagger} \mathbf{v}_{\ddagger}^{2}+h^{-3} \mathbf{c}^{\prime}\left(-\beta^{-1} \nabla_{\ddagger} \cdot \beta \nabla_{\ddagger} v_{3}^{1}+\beta^{-1} \mathbf{c}^{\prime} \partial_{s} p^{0}\right) .
\end{aligned}
$$

Applying (37) to the above, we obtain

$$
\mathbf{F}^{h}=-h^{-2} X^{h} \beta^{-1} \nabla_{\ddagger} \cdot \beta \nabla_{\ddagger} \mathbf{v}_{\ddagger}^{2}-h^{-1} \beta^{-1} \partial_{s} \beta^{-1} \partial_{s}\left(\mathbf{c}^{\prime} v_{3}^{1}\right)-\beta^{-1} \partial_{s} \beta^{-1} \partial_{s}\left(X^{h} \mathbf{v}_{\ddagger}^{2}\right) .
$$

Now, let us consider the differences $\tilde{\mathbf{v}}^{h}=\mathbf{v}^{h}-\mathbf{v}^{h}-\mathbf{w}$ and $p_{\text {rem }}^{h}=p^{h}-p^{h}$ between the true and approximate solutions. The vector $\tilde{\mathbf{v}}^{h}$ is solenoidal by construction. Then, integration by parts and (56) give us

$$
\begin{aligned}
& \left(\nabla_{\mathbf{x}} \tilde{\mathbf{v}}^{h}+\nabla_{\mathbf{x}} \mathbf{w}, \nabla_{\mathbf{x}} \tilde{\mathbf{v}}^{h}\right)=\left\|\nabla_{\mathbf{x}} \tilde{\mathbf{v}}^{h}\right\|^{2}+\left(\nabla_{\mathbf{x}} \mathbf{w}, \nabla_{\mathbf{x}} \tilde{\mathbf{v}}^{h}\right) \\
& \quad=\int_{\omega^{h}(1)}\left(\beta^{-1} \partial_{s}\left(v_{3}^{h}-\nabla_{3}^{h}\right)-p^{h}+\mathbb{p}^{h}\right) \tilde{v}_{3}^{h} \mathrm{~d} \sigma(r, \theta)-\left(\mathbf{F}^{h}, \tilde{\mathbf{v}}^{h}\right) \\
& =-\int_{\omega^{h}(1)} h^{-1} \beta^{-1}\left(\partial_{s} v_{3}^{1}\right) \tilde{v}_{3}^{h} \mathrm{~d} \sigma(r, \theta)-\left(\mathbf{F}^{h}, \tilde{\mathbf{v}}^{h}\right) .
\end{aligned}
$$

Substituting the expression for $\mathbf{F}^{h}$, the equation above can be written as

$$
\begin{aligned}
\left\|\nabla_{\mathbf{x}} \tilde{\mathbf{v}}^{h}\right\|^{2}= & -\left(\nabla_{\mathbf{x}} \mathbf{w}, \nabla_{\mathbf{x}} \tilde{\mathbf{v}}^{h}\right)+\left(h^{-2} X^{h} \beta^{-1} \nabla_{\ddagger} \cdot \beta \nabla_{\ddagger} \mathbf{v}_{\ddagger}^{2}, \tilde{\mathbf{v}}^{h}\right) \\
& \int_{\omega^{h}(1)} h^{-1} \beta^{-1}\left(\partial_{s} v_{3}^{1}\right) \tilde{v}_{3}^{h} \mathrm{~d} \sigma(r, \theta)+\left(\beta^{-1} \partial_{s} \beta^{-1} \partial_{s}\left(h^{-1} \mathbf{c}^{\prime} v_{3}^{1}+X^{h} \mathbf{v}_{\ddagger}^{2}\right), \tilde{\mathbf{v}}^{h}\right)
\end{aligned}
$$

Integrating by parts again and using the fact that $\tilde{\mathbf{v}}^{h}$ is divergence-free, we get

$$
\begin{aligned}
& \left\|\nabla_{\mathbf{x}} \tilde{\mathbf{v}}^{h}\right\|^{2}=-h^{-1}\left(\beta^{-1} v_{3}^{1} \mathbf{c}^{\prime \prime}, \beta^{-1} \partial_{s} \tilde{\mathbf{v}}^{h}\right)-h^{-1}\left(\beta^{-1} \mathbf{c}^{\prime} \partial_{s}\left(v_{3}^{1}\right), \beta^{-1} \partial_{s} \tilde{\mathbf{v}}^{h}\right) \\
& -h^{-1}\left(X^{h} \nabla_{\ddagger} \mathbf{v}_{\ddagger}^{2}, \nabla_{\bullet} \tilde{\mathbf{v}}^{h}\right)-\left(\beta^{-1} \partial_{s}\left(X^{h} \mathbf{v}_{\ddagger}^{2}\right), \beta^{-1} \partial_{s} \tilde{\mathbf{v}}^{h}\right)-\left(\nabla_{\mathbf{x}} \mathbf{w}, \nabla_{\mathbf{x}} \tilde{\mathbf{v}}^{h}\right) .
\end{aligned}
$$

Let us now estimate the terms in (60) by using the previously derived estimates. At this point, we shall assume that the parameters $\lambda, \gamma, \lambda^{*}$ and $\gamma^{*}$ are such that

$$
\gamma=O(\lambda), \quad \gamma^{*}=O\left(\lambda^{*}\right), \quad \text { and } \quad \lambda^{*}=O\left(h^{-1 / 2} \lambda^{3 / 2}\right) .
$$

With the help of (50) and (13), the first term on the right hand side of (60) can be estimated as

$$
h^{-1}\left|\left(\beta^{-1} v_{3}^{1} \mathbf{c}^{\prime \prime}, \beta^{-1} \partial_{s} \tilde{\mathbf{v}}^{h}\right)\right| \leq c h^{-1} h h^{-1 / 2} \lambda^{1 / 2}\left\|\partial_{s} \tilde{\mathbf{v}}^{h}\right\| \leq c h^{-1 / 2} \lambda^{1 / 2}\left\|\nabla_{\mathbf{x}} \tilde{\mathbf{v}}^{h}\right\| .
$$

Using (50) to estimate the second term, we have

$$
h^{-1}\left|\left(\beta^{-1} \mathbf{c}^{\prime} \partial_{s}\left(v_{3}^{1}\right), \beta^{-1} \partial_{s} \tilde{\mathbf{v}}^{h}\right)\right| \leq c h^{-1} h \lambda\left\|\partial_{s} \tilde{\mathbf{v}}^{h}\right\| \leq c h^{0} \lambda\left\|\nabla_{\mathbf{x}} \tilde{\mathbf{v}}^{h}\right\| .
$$

We use (51) to get

$$
h^{-1}\left|\left(X^{h} \nabla_{\ddagger} \mathbf{v}_{\ddagger}^{2}, \nabla \cdot \tilde{\mathbf{v}}^{h}\right)\right| \leq c h^{-1} h \lambda\left\|\nabla \cdot \tilde{\mathbf{v}}^{h}\right\| \leq c h^{0} \lambda\left\|\nabla_{\mathbf{x}} \tilde{\mathbf{v}}^{h}\right\| .
$$

Then due to (52) and (13), we have

$$
\left|\left(\beta^{-1} \partial_{s}\left(X^{h} \mathbf{v}_{\ddagger}^{2}\right), \beta^{-1} \partial_{s} \tilde{\mathbf{v}}^{h}\right)\right| \leq c h^{1 / 2} \lambda^{3 / 2}\left\|\partial_{s} \tilde{\mathbf{v}}^{h}\right\| \leq c h^{1 / 2} \lambda^{3 / 2}\left\|\nabla_{\mathbf{x}} \tilde{\mathbf{v}}^{h}\right\| .
$$

Finally, (59) gives us

$$
\left|\left(\nabla_{\mathbf{x}} \mathbf{w}, \nabla_{\mathbf{x}} \tilde{\mathbf{v}}^{h}\right)\right| \leq c h^{1 / 2} \lambda\left\|\nabla_{\mathbf{x}} \tilde{\mathbf{v}}^{h}\right\|
$$

Also, by Friedrichs's inequality,

$$
\left\|\tilde{\mathbf{v}}^{h}\right\| \leq \operatorname{ch}\left\|\nabla_{\mathbf{x}} \tilde{\mathbf{v}}^{h}\right\|
$$

for the curved cylinder $\Omega^{h}$. Thus, for the discrepancy in the velocity, we arrive at the estimate

$$
\left\|\nabla_{\mathbf{x}} \tilde{\mathbf{v}}^{h}\right\|+h^{-1}\left\|\tilde{\mathbf{v}}^{h}\right\| \leq c h^{-1 / 2} \lambda^{1 / 2} .
$$


Here we note that $\left\|\nabla_{\mathbf{x}} \mathbf{w}\right\|$ is $O\left(h^{1 / 2} \lambda\right)$ while $\left\|\nabla_{\mathbf{x}} \tilde{\mathbf{v}}^{h}\right\|$ is $O\left(h^{-1 / 2} \lambda^{1 / 2}\right)$ which leads to

$$
\left\|\nabla_{\mathbf{x}} \mathbf{v}_{\text {rem }}^{h}\right\| \leq\left\|\nabla_{\mathbf{x}} \tilde{\mathbf{v}}^{h}\right\|+\left\|\nabla_{\mathbf{x}} \mathbf{w}\right\| \leq c h^{-1 / 2} \lambda^{1 / 2} .
$$

Moreover, as $\|\mathbf{w}\|$ is $O\left(h^{3 / 2} \lambda\right)$ and $\left\|\tilde{\mathbf{v}}^{h}\right\|$ is $O\left(h^{1 / 2} \lambda^{1 / 2}\right)$,

$$
\left\|\mathbf{v}_{r e m}^{h}\right\| \leq\left\|\tilde{\mathbf{v}}^{h}\right\|+\|\mathbf{w}\| \leq \operatorname{ch}^{1 / 2} \lambda^{1 / 2} .
$$

On the other hand, $\left\|\nabla_{\mathbf{x}} \mathbf{v}^{h}\right\|$ is $O\left(h^{-1}\right)$ whereas $\left\|\mathbf{v}^{h}\right\|$ is $O\left(h^{0}\right)$. Thus it is safe to conclude that the approximation of velocity is justified for a $\lambda$ which is $O\left(h^{-1+2 \delta}\right)$ for any $\delta>0$.

Let us now estimate the discrepancy in the approximation of pressure. Let us denote the average of a scalar field over $\Omega^{h}$ by placing a bar over the corresponding symbol. Consider the velocity field $\tilde{\mathbf{w}}$ such that

$$
\begin{aligned}
-\nabla_{\mathbf{x}} \cdot \tilde{\mathbf{w}} & =p_{r e m}^{h}-\bar{p}_{r e m}^{h} & & \text { in } \Omega^{h}, \\
\tilde{\mathbf{w}} & =\mathbf{0} & & \text { on } \partial \Omega^{h} .
\end{aligned}
$$

Clearly, the compatibility condition (30) is satisfied.

Then, integration by parts and equation (56) result in

$$
\begin{aligned}
& \left(\nabla_{\mathbf{x}} \tilde{\mathbf{v}}^{h}+\nabla_{\mathbf{x}} \mathbf{w}, \nabla_{\mathbf{x}} \tilde{\mathbf{w}}\right)+\left\|p_{r e m}^{h}-\bar{p}_{r e m}^{h}\right\|^{2} \\
& =\int_{\omega^{h}(1)}\left(\beta^{-1} \partial_{s}\left(v_{3}^{h}-\nabla_{3}^{h}+w_{3}\right)-p^{h}+\mathbb{p}^{h}\right) \tilde{w}_{3} \mathrm{~d} \sigma(r, \theta)-\left(\mathbf{F}^{h}, \tilde{\mathbf{w}}\right) \\
& =-\int_{\omega^{h}(1)} h^{-1}\left(\beta^{-1} \partial_{s} v_{3}^{1}\right) \tilde{w}_{3} \mathrm{~d} \sigma(r, \theta)-\left(\mathbf{F}^{h}, \tilde{\mathbf{w}}\right) .
\end{aligned}
$$

Similar steps as before lead us to

$$
\begin{aligned}
\left\|p_{r e m}^{h}-\bar{p}_{\text {rem }}^{h}\right\|^{2}= & -\left(\nabla_{\mathbf{x}} \tilde{\mathbf{v}}^{h}, \nabla_{\mathbf{x}} \tilde{\mathbf{w}}\right)-\left(\nabla_{\mathbf{x}} \mathbf{w}, \nabla_{\mathbf{x}} \tilde{\mathbf{w}}\right) \\
& -h^{-1}\left(\beta^{-1} v_{3}^{1} \mathbf{c}^{\prime \prime}, \beta^{-1} \partial_{s} \tilde{\mathbf{w}}\right)-h^{-1}\left(\beta^{-1} \mathbf{c}^{\prime} \partial_{s}\left(v_{3}^{1}\right), \beta^{-1} \partial_{s} \tilde{\mathbf{w}}\right) \\
& -h^{-1}\left(X^{h} \nabla_{\ddagger} \mathbf{v}_{\ddagger}^{2}, \nabla \cdot \tilde{\mathbf{w}}\right)-\left(\beta^{-1} \partial_{s}\left(X^{h} \mathbf{v}_{\ddagger}^{2}\right), \beta^{-1} \partial_{s} \tilde{\mathbf{w}}\right) .
\end{aligned}
$$

Using (61) and Lemma 1, we estimate the first term on the right hand side of (62) as

$$
\left|\left(\nabla_{\mathbf{x}} \tilde{\mathbf{v}}^{h}, \nabla_{\mathbf{x}} \tilde{\mathbf{w}}\right)\right| \leq c h^{-1 / 2} \lambda^{1 / 2}\left\|\nabla_{\mathbf{x}} \tilde{\mathbf{w}}\right\| \leq c h^{-3 / 2} \lambda^{1 / 2}\left\|p_{r e m}^{h}-\bar{p}_{r e m}^{h}\right\| .
$$

Due to (59), for the next term, we have

$$
\left|\left(\nabla_{\mathbf{x}} \mathbf{w}, \nabla_{\mathbf{x}} \tilde{\mathbf{w}}\right)\right| \leq c h^{1 / 2} \lambda\left\|\nabla_{\mathbf{x}} \tilde{\mathbf{w}}\right\| \leq c h^{-1 / 2} \lambda^{1 / 2}\left\|p_{r e m}^{h}-\bar{p}_{\text {rem }}^{h}\right\| .
$$

Once again by Lemma $1,(50)$ and (13), we get

$$
\begin{gathered}
\left|h^{-1}\left(\beta^{-1} v_{3}^{1} \mathbf{c}^{\prime \prime}, \beta^{-1} \partial_{s} \tilde{\mathbf{w}}\right)\right| \leq c h^{-1} h h^{-1 / 2} \lambda^{1 / 2}\left\|\nabla_{\mathbf{x}} \tilde{\mathbf{w}}\right\| \leq c h^{-3 / 2} \lambda^{1 / 2}\left\|p_{r e m}^{h}-\bar{p}_{r e m}^{h}\right\|, \\
\left|h^{-1}\left(\beta^{-1} \mathbf{c}^{\prime} \partial_{s}\left(v_{3}^{1}\right), \beta^{-1} \partial_{s} \tilde{\mathbf{w}}\right)\right| \leq c h^{-1} h \lambda\left\|\nabla_{\mathbf{x}} \tilde{\mathbf{w}}\right\| \leq c h^{-1} \lambda\left\|p_{r e m}^{h}-\bar{p}_{r e m}^{h}\right\| .
\end{gathered}
$$

We use (51) to obtain

$$
h^{-1}\left|\left(X^{h} \nabla_{\ddagger} \mathbf{v}_{\ddagger}^{2}, \nabla \cdot \tilde{\mathbf{w}}\right)\right| \leq c h^{-1} h \lambda\left\|\nabla_{\mathbf{x}} \tilde{\mathbf{w}}\right\| \leq c h^{-1} \lambda\left\|p_{r e m}^{h}-\bar{p}_{\text {rem }}^{h}\right\| .
$$

Then due to (52) and (13), we have

$$
\left|\left(\beta^{-1} \partial_{s}\left(X^{h} \mathbf{v}_{\ddagger}^{2}\right), \beta^{-1} \partial_{s} \tilde{\mathbf{w}}\right)\right| \leq c h^{1 / 2} \lambda^{3 / 2}\left\|\nabla_{\mathbf{x}} \tilde{\mathbf{w}}\right\| \leq c h^{-1 / 2} \lambda^{3 / 2}\left\|p_{r e m}^{h}-\bar{p}_{r e m}^{h}\right\| .
$$

Thus, for the discrepancy in the pressure, we arrive at

$$
\left\|p_{\text {rem }}^{h}-\bar{p}_{\text {rem }}^{h}\right\| \leq c h^{-3 / 2} \lambda^{1 / 2} .
$$

As $\left\|p^{h}-\bar{p}^{h}\right\|$ is $O\left(h^{-2}\right)$, once again we see that the approximate pressure upto a constant is justified for a $\lambda$ which is $O\left(h^{-1+2 \delta}\right)$ for any $\delta>0$.

To summarize, we have shown that (7) and (8) hold under the assumptions (5) thereby justifying our asymptotic approximations. 


\section{The Case of $O(1)$ Curvature}

The more conventional method to tackle the problem in the case of a mildly curving pipe, where we assume that $\mathbf{c}$ is a smooth function whose derivatives are bounded independently of $h$, would be to expand the scale factor $\beta$ as $1-h \eta \mathbf{c}^{\prime \prime} \cdot \mathbf{e}_{1}$, instead of keeping it as a parameter for the asymptotic procedure. Let us compare the results obtained by our method in this case with those obtained with the conventional method as mentioned. For this case, the assumptions on the geometry of the centre curve are such that

$$
\left|\mathbf{c}^{\prime \prime \prime}\right| \leq c h^{0}, \quad\left|\mathbf{c}^{\prime \prime \prime \prime}\right| \leq c h^{0}, \quad\left|\partial_{s} R\right| \leq c h^{0} \quad \text { and } \quad\left|\partial_{s}^{2} R\right| \leq c h^{0} .
$$

The first inequality above implies that the curvature has the restriction

$$
\left|\mathbf{c}^{\prime \prime}\right| \leq c h^{0} .
$$

With these assumptions, let the solution $\left\{\mathbf{v}^{h}, p^{h}\right\}$ admit the following formal asymptotic expansions due to the conventional method:

$$
\begin{gathered}
p^{h}=h^{-3} q^{0}+h^{-2} q^{1}+\cdots, \\
\mathbf{v}^{h}=h^{-1} \mathbf{u}^{1}+\mathbf{u}^{2}+\cdots .
\end{gathered}
$$

Then, (1), (2) and (3) imply that $u_{3}^{1}$ and $q^{0}=q^{0}(s)$ satisty

$$
\begin{gathered}
-\Delta_{\ddagger} u_{3}^{1}+\partial_{s} q^{0}=0 \text { in } \omega(s), \\
u_{3}^{1}=0 \text { on } \partial \omega(s) .
\end{gathered}
$$

where as $u_{3}^{2}$ and $q^{1}$ fulfill

$$
\begin{gathered}
-\Delta_{\ddagger} u_{3}^{2}+\partial_{s} q^{1}=-\mathbf{c}^{\prime \prime} \cdot \nabla_{\ddagger} u_{3}^{1}-\eta \mathbf{c}^{\prime \prime} \cdot \mathbf{e}_{1} \partial_{s} q^{0} \text { in } \omega(s), \\
u_{3}^{2}=0 \text { on } \partial \omega(s) .
\end{gathered}
$$

It can be shown that $\mathbf{u}_{\ddagger}^{1}$ is still $\mathbf{0}_{\ddagger}$ as well as $q^{1}=q^{1}(s)$. One can obtain $\mathbf{u}_{\ddagger}^{2}$ and $q^{2}$ from

$$
\begin{gathered}
-\Delta_{\ddagger} \mathbf{u}_{\ddagger}^{2}+\nabla_{\ddagger} q^{2}=\mathbf{0}_{\ddagger}, \quad-\nabla_{\ddagger} \cdot \mathbf{u}_{\ddagger}^{2}=\partial_{s} u_{3}^{1} \quad \text { in } \quad \omega(s), \\
\mathbf{u}_{\ddagger}^{2}=\mathbf{0}_{\ddagger} \quad \text { on } \quad \partial \omega(s)
\end{gathered}
$$

whereas, for the next terms, we have

$$
\begin{gathered}
-\Delta_{\ddagger} \mathbf{u}_{\ddagger}^{3}+\nabla_{\ddagger} q^{3}=\mathbf{c}_{\ddagger}^{\prime \prime \prime} u_{3}^{1}+2 \mathbf{c}^{\prime \prime} \partial_{s} u_{3}^{1}-\mathbf{c}^{\prime \prime} \cdot \nabla_{\ddagger} \mathbf{u}_{\ddagger}^{2}, \\
-\nabla_{\ddagger} \cdot \mathbf{u}_{\ddagger}^{3}=\partial_{s} u_{3}^{2}-\mathbf{c}^{\prime \prime} \cdot \mathbf{u}_{\ddagger}^{2}+\eta \mathbf{c}^{\prime \prime} \cdot \mathbf{e}_{1} \partial_{s} u_{3}^{1} \text { in } \omega(s), \\
\mathbf{u}_{\ddagger}^{3}=\mathbf{0}_{\ddagger} \text { on } \partial \omega(s) .
\end{gathered}
$$

Due to (63), we obtain

$$
u_{3}^{1}=-\frac{1}{2} \Psi_{0} \partial_{s} q^{0}
$$

where the Prandtl function $\Psi_{0}$ is the solution of

$$
-\Delta_{\ddagger} \Psi_{0}=2 \quad \text { in } \omega(s), \quad \Psi_{0}=0 \quad \text { on } \partial \omega(s) .
$$

Similarly, (64) gives

$$
u_{3}^{2}=-\frac{1}{2}\left(\Psi_{1} \partial_{s} q^{0}+\Psi_{0} \partial_{s} q^{1}\right)
$$

where the function $\Psi_{1}$ is the solution of

$$
-\Delta_{\ddagger} \Psi_{1}=2 \eta \mathbf{c}^{\prime \prime} \cdot \mathbf{e}_{1}-\mathbf{c}^{\prime \prime} \cdot \nabla_{\ddagger} \Psi_{0} \quad \text { in } \quad \omega(s), \quad \Psi_{1}=0 \quad \text { on } \quad \partial \omega(s) .
$$


For the discrepancy in approximating the function $\Psi$ obtained by our method with $\Psi_{0}+h \Psi_{1}$ we find using (39), (67) and (68) that

$$
\begin{aligned}
& -\beta^{-1} \nabla_{\ddagger} \cdot \beta \nabla_{\ddagger}\left(\Psi-\Psi_{0}-h \Psi_{1}\right)=-\Delta_{\ddagger}\left(\Psi-\Psi_{0}-h \Psi_{1}\right)+h \beta^{-1} \mathbf{c}^{\prime \prime} \cdot \nabla_{\ddagger}\left(\Psi-\Psi_{0}-h \Psi_{1}\right) \\
& \quad=2 \beta^{-1}-2-h\left(2 \eta \mathbf{c}^{\prime \prime} \cdot \mathbf{e}_{1}-\mathbf{c}^{\prime \prime} \cdot \nabla_{\ddagger} \Psi_{0}\right)-h \beta^{-1} \mathbf{c}^{\prime \prime} \cdot \nabla_{\ddagger} \Psi_{0}-h^{2} \beta^{-1} \mathbf{c}^{\prime \prime} \cdot \nabla_{\ddagger} \Psi_{1} \\
& \quad=2\left(\beta^{-1}-1-h \eta \mathbf{c}^{\prime \prime} \cdot \mathbf{e}_{1}\right)-h^{2} \beta^{-1} \mathbf{c}^{\prime \prime} \cdot \nabla_{\ddagger} \Psi_{1}=O\left(h^{2}\right) .
\end{aligned}
$$

Thus we conclude that $\Psi_{0}+h \Psi_{1}$ approximates $\Psi$ up to order $h^{2}$. It follows that, defining

$$
G_{i}(s):=2 \int_{\omega(s)} \Psi_{i}(\eta, \theta, s) \mathrm{d} \sigma(\eta, \theta), \quad i \in\{0,1\},
$$

we get an approximation $G_{0}+h G_{1}$ for the function $G$ with error $O\left(h^{2}\right)$.

Note that due to the boundary and the divergence conditions in (65),

$$
\begin{aligned}
& \int_{\omega(s)}\left(\mathbf{c}^{\prime \prime} \cdot \mathbf{u}_{\ddagger}^{2}-\eta \mathbf{c}^{\prime \prime} \cdot \mathbf{e}_{1} \partial_{s} u_{3}^{1}\right) \mathrm{d} \sigma(\eta, \theta)=\int_{\omega(s)}\left(\mathbf{c}^{\prime \prime} \cdot \mathbf{u}_{\ddagger}^{2}+\eta \mathbf{c}^{\prime \prime} \cdot \mathbf{e}_{1} \nabla_{\ddagger} \cdot \mathbf{u}_{\ddagger}^{2}\right) \mathrm{d} \sigma(\eta, \theta) \\
& \quad=\int_{\omega(s)} \nabla_{\ddagger} \cdot\left(\eta \mathbf{c}^{\prime \prime} \cdot \mathbf{e}_{1} \mathbf{u}_{\ddagger}^{2}\right) \mathrm{d} \sigma(\eta, \theta)=0 .
\end{aligned}
$$

Hence, the compatibility conditions in (65) and (66) respectively provide the equations for $q^{0}$ and $q^{1}$ as

$$
-\partial_{s}\left(G_{0}(s) \partial_{s} q^{0}(s)\right)=0 \quad \text { and } \quad-\partial_{s}\left(G_{0}(s) \partial_{s} q^{1}(s)+G_{1}(s) \partial_{s} q^{0}(s)\right)=0, \quad s \in(0,1) .
$$

We use the same boundary conditions as in (48) and (49) so that

$$
\begin{aligned}
& -G_{0}(0) \partial_{s} q^{0}(0)=4 F^{0} \quad \text { and } \quad q^{0}(1)=p_{p e r}^{0}, \\
& -G_{0}(0) \partial_{s} q^{1}(0)-G_{1}(0) \partial_{s} q^{0}(0)=0 \quad \text { and } \quad q^{1}(1)=0 \text {. }
\end{aligned}
$$

Thus we have the solutions

$$
\begin{aligned}
& q^{0}(s)=p_{\text {per }}^{0}+4 F^{0} \int_{s}^{1} \frac{1}{G_{0}(t)} \mathrm{d} t, \\
& q^{1}(s)=-4 F^{0} \int_{s}^{1} \frac{G_{1}(t)}{G_{0}(t)^{2}} \mathrm{~d} t .
\end{aligned}
$$

Then for the discrepancy in the approximations in pressure, we have

$$
\begin{gathered}
p^{0}(s)-q^{0}(s)-h q^{1}(s)=4 F^{0} \int_{s}^{1}\left(\frac{1}{G(t)}-\frac{1}{G_{0}(t)}\left(1-\frac{h G_{1}(t)}{G_{0}(t)}\right)\right) \mathrm{d} t \\
=4 F^{0} \int_{s}^{1}\left(\frac{1}{G(t)}-\frac{1}{G_{0}(t)+h G_{1}(t)}+O\left(h^{2}\right)\right) \mathrm{d} t=O\left(h^{2}\right) .
\end{gathered}
$$

Now let us consider the difference in the velocity components given by the two methods. For the longitudinal part, we have

$$
\begin{aligned}
& 2\left|v_{3}^{1}-u_{3}^{1}-h u_{3}^{2}\right|=\left|\Psi \partial_{s} p^{0}-\Psi_{0} \partial_{s} q^{0}-h\left(\Psi_{0} \partial_{s} q^{1}+\Psi_{1} \partial_{s} q^{0}\right)\right| \\
& \left.\quad=\mid\left(\Psi_{0}+h \Psi_{1}\right) \partial_{s}\left(q^{0}+h q^{1}\right)+O\left(h^{2}\right)-\left(\Psi_{0}+h \Psi_{1}\right) \partial_{s} q^{0}-h \Psi_{0} \partial_{s} q^{1}\right) \mid=O\left(h^{2}\right) .
\end{aligned}
$$


On the other hand, for the transversal components, we consider (65), (66) and (46) so that

$$
\begin{aligned}
- & \beta^{-1} \nabla_{\ddagger} \cdot \beta \nabla_{\ddagger}\left(\mathbf{v}_{\ddagger}^{2}-\mathbf{u}_{\ddagger}^{2}-h \mathbf{u}_{\ddagger}^{3}\right)+\nabla_{\ddagger}\left(p^{2}-q^{2}-h q^{3}\right) \\
& =h \mathbf{c}_{\ddagger}^{\prime \prime \prime}\left(\beta^{-2} v_{3}^{1}-u_{3}^{1}\right)+h c^{\prime \prime}\left(2 \beta^{-2} \partial_{s} v_{3}^{1}-2 \partial_{s} u_{3}^{1}-\beta^{-3} v_{3}^{1} \partial_{s} \beta\right) \\
& =O\left(h^{2}\right) \mathbf{e}_{1}+O\left(h^{2}\right) \mathbf{e}_{2},
\end{aligned}
$$

as well as

$$
\begin{aligned}
& -\nabla_{\ddagger} \cdot \beta\left(\mathbf{v}_{\ddagger}^{2}-\mathbf{u}_{\ddagger}^{2}-h \mathbf{u}_{\ddagger}^{3}\right)=\partial_{s} v_{3}^{1}-\beta\left(\partial_{s} u_{3}^{1}+h \partial_{s} u_{3}^{2}\right) \\
& +h \beta\left(\mathbf{c}^{\prime \prime} \cdot \mathbf{u}_{\ddagger}^{2}-\eta \mathbf{c}^{\prime \prime} \cdot \mathbf{e}_{1} \partial_{s} u_{3}^{1}\right)-h \mathbf{c}^{\prime \prime} \cdot\left(\mathbf{u}_{\ddagger}^{2}+h \mathbf{u}_{\ddagger}^{2}\right) \\
& =\partial_{s} v_{3}^{1}-\partial_{s} u_{3}^{1}-h \partial_{s} u_{3}^{2}+h(\beta-1)\left(\mathbf{c}^{\prime \prime} \cdot \mathbf{u}_{\ddagger}^{2}-\partial_{s} u_{3}^{2}\right)+(\beta-1)^{2} \partial_{s} u_{3}^{1}=O\left(h^{2}\right) .
\end{aligned}
$$

Thus, we conclude that our method produces two-term asymptotic approximations corresponding to a more conventional method for the solution of the problem (1), (2) and (3) in the case of mild curvature.

Funding Open access funding provided by Linköping University.

\section{Compliance with Ethical Standards}

Conflict of interest On behalf of all authors, the corresponding author states that there is no conflict of interest.

Open Access. This article is licensed under a Creative Commons Attribution 4.0 International License, which permits use, sharing, adaptation, distribution and reproduction in any medium or format, as long as you give appropriate credit to the original author(s) and the source, provide a link to the Creative Commons licence, and indicate if changes were made. The images or other third party material in this article are included in the article's Creative Commons licence, unless indicated otherwise in a credit line to the material. If material is not included in the article's Creative Commons licence and your intended use is not permitted by statutory regulation or exceeds the permitted use, you will need to obtain permission directly from the copyright holder. To view a copy of this licence, visit http://creativecommons.org/licenses/by/4.0/.

Publisher's Note Springer Nature remains neutral with regard to jurisdictional claims in published maps and institutional affiliations.

\section{Appendix A. Proof of Claim 1}

Proof. Firstly, note that the normal $R \mathbf{e}_{1}-\left(\partial_{\theta} R\right) \mathbf{e}_{2}=-\partial_{\theta}\left(R \mathbf{e}_{2}\right)$. Then due to the divergence theorem and (21),

$$
-\int_{\omega(s)} \nabla_{\ddagger} \cdot\left(\partial_{s} \beta\right) \mathbf{u} \mathrm{d} \sigma(\eta, \theta)=\left.\int_{0}^{2 \pi}\left(\left(\partial_{s} \beta\right) \mathbf{u}\right)\right|_{\eta=R} \cdot \partial_{\theta}\left(R \mathbf{e}_{2}\right) \mathrm{d} \theta=\left.\int_{0}^{2 \pi}\left(\partial_{s} \beta\right)\right|_{\eta=R} \mathbf{h} \cdot \partial_{\theta}\left(R \mathbf{e}_{2}\right) \mathrm{d} \theta .
$$

Once again due to (21),

$$
-\int_{\omega(s)} \beta^{-1}\left(\partial_{s} \beta\right) \nabla_{\ddagger} \cdot \beta \mathbf{u} \mathrm{d} \sigma(\eta, \theta)=\int_{\omega(s)}\left(\partial_{s} \beta\right) g \mathrm{~d} \sigma(\eta, \theta) .
$$

On the other hand, deriving (20) with respect to $s$ leads us to

$$
\begin{aligned}
0 & =\int_{\omega(s)}\left(\left(\partial_{s} \beta\right) g+\beta \partial_{s} g\right) \mathrm{d} \sigma(\eta, \theta)+\left.\int_{0}^{2 \pi}\left(\partial_{s} R\right)(\beta g)\right|_{\eta=R} R \mathrm{~d} \theta \\
& -\left.\int_{0}^{2 \pi}\left(\left.\left(\beta \partial_{s} \mathbf{h}+\left(\partial_{s} \beta\right) \mathbf{h}+\left(\partial_{\eta} \beta\right)\left(\partial_{s} R\right) \mathbf{h}\right)\right|_{\eta=R} \cdot \partial_{\theta}\left(R \mathbf{e}_{2}\right)+\beta \mathbf{h} \cdot \partial_{s} \partial_{\theta}\left(R \mathbf{e}_{2}\right)\right)\right|_{\eta=R} \mathrm{~d} \theta .
\end{aligned}
$$


Hence, to prove the claim, it suffices to show that

$$
\left.\int_{0}^{2 \pi}\left(\left(\partial_{s} R\right)\left(\left(\beta \partial_{\eta} \mathbf{u}+\left(\partial_{\eta} \beta\right) \mathbf{h}\right) \cdot \partial_{\theta}\left(R \mathbf{e}_{2}\right)-R \beta g\right)+\beta \mathbf{h} \cdot \partial_{s} \partial_{\theta}\left(R \mathbf{e}_{2}\right)\right)\right|_{\eta=R} \mathrm{~d} \theta=0 .
$$

Considering the first term under the integral above, we can use the expansion

$$
\beta \partial_{\eta} \mathbf{u} \cdot \partial_{\theta}\left(R \mathbf{e}_{2}\right)=\beta\left(\left(\partial_{\theta} R\right) \partial_{\eta} u_{2}-R \partial_{\eta} u_{1}\right) .
$$

Then for the next term, due to (21) and the fact that $\mathbf{e}_{1} \cdot \nabla_{\ddagger}=\partial_{\eta}$, one has

$$
\left(\partial_{\eta} \beta\right) \mathbf{h} \cdot \partial_{\theta}\left(R \mathbf{e}_{2}\right)=\mathbf{e}_{1} \cdot \nabla_{\ddagger} \beta\left(\left(\partial_{\theta} R\right) u_{2}-R u_{1}\right) .
$$

For the third term, we have

$$
-R \beta g=R \nabla_{\ddagger} \cdot \beta \mathbf{u}=\beta\left(R \partial_{\eta} u_{1}+u_{1}+\partial_{\theta} u_{2}\right)+R\left(u_{1} \mathbf{e}_{1}+u_{2} \mathbf{e}_{2}\right) \cdot \nabla_{\ddagger} \beta .
$$

Lastly, noting that $\eta \mathbf{e}_{2} \cdot \nabla_{\ddagger}=\partial_{\theta}$, we obtain

$$
\begin{aligned}
& \left.\int_{0}^{2 \pi} \beta\right|_{\eta=R} \mathbf{h} \cdot \partial_{s} \partial_{\theta}\left(R \mathbf{e}_{2}\right) \mathrm{d} \theta=-\int_{0}^{2 \pi} \partial_{\theta}\left(\left.(\beta \mathbf{u})\right|_{\eta=R}\right) \cdot \partial_{s}\left(R \mathbf{e}_{2}\right) \mathrm{d} \theta \\
& =-\int_{0}^{2 \pi}\left(\partial_{s} R\right)\left(\beta\left(u_{1}+\partial_{\theta} u_{2}+\left(\partial_{\theta} R\right) \partial_{\eta} u_{2}\right)+u_{2}\left(\mathbf{e}_{1} \partial_{\theta} R+\mathbf{e}_{2} R\right) \cdot \nabla_{\ddagger} \beta\right) \mathrm{d} \theta .
\end{aligned}
$$

Combining the above, the claim is proved.

\section{References}

[1] Bourgeat, A., Marušić-Paloka, E.: Nonlinear effects for flow in periodically constricted channel caused by high injection rate. Math. Models Methods Appl. Sci. 8(3), 379-405 (1998)

[2] Čanić, S., Mikelić, A.: Effective equations modeling the flow of a viscous incompressible fluid through a long elastic tube arising in the study of blood flow through small arteries. SIAM J. Appl. Dyn. Syst. 2(3), 431-463 (2003)

[3] Dupuy, D., Panasenko, G.P., Stavre, R.: Asymptotic solution for a micropolar flow in a curvilinear channel. ZAMM Z. Angew. Math. Mech. 88(10), 793-807 (2008)

[4] Galdi, G.P.: An introduction to the mathematical theory of the Navier-Stokes equations, vol. I, volume 38 of Springer Tracts in Natural Philosophy. Linearized Steady Problems. Springer, New York (1994)

[5] Huy, N.D., Stará, J.: On existence and regularity of solutions to a class of generalized stationary Stokes problem. Comment. Math. Univ. Carolin. 47(2), 241-264 (2006)

[6] Ladyženskaja, O.A., Solonnikov, V.A.: Some problems of vector analysis, and generalized formulations of boundary value problems for the Navier-Stokes equation. Zap. Naučn. Sem. Leningrad. Otdel. Mat. Inst. Steklov. (LOMI), 59, 81-116, 256 (1976). Boundary value problems of mathematical physics and related questions in the theory of functions, 9

[7] Ladyzhenskaya, O.A.: The mathematical theory of viscous incompressible flow. Second English edition, revised and enlarged. Translated from the Russian by Richard A. Silverman and John Chu. Mathematics and its Applications, vol. 2. Gordon and Breach. Science Publishers, New York (1969)

[8] Lions, J.-L., Magenes, E.: Non-homogeneous boundary value problems and applications, vol. I. Springer, New YorkHeidelberg. Translated from the French by P, p. 181. Kenneth, Die Grundlehren der mathematischen Wissenschaften, Band (1972)

[9] Littman, W., Stampacchia, G., Weinberger, H.F.: Regular points for elliptic equations with discontinuous coefficients. Ann. Scuola Norm. Sup. Pisa 3(17), 43-77 (1963)

[10] Marušić-Paloka, E.: The effects of flexion and torsion on a fluid flow through a curved pipe. Appl. Math. Optim. 44(3), $245-272(2001)$

[11] Nazarov, S.A.: Asymptotic solution of the Navier-Stokes problem on the flow of a thin layer of fluid. Sibirsk. Mat. Zh. 31(2), 131-144 (1990)

[12] Nazarov, S.A., Piletskas, K.I.: The Reynolds flow of a fluid in a thin three-dimensional channel. Litovsk. Mat. Sb. 30(4), $772-783(1990)$

[13] Panasenko, G., Pileckas, K.: Asymptotic analysis of the non-steady Navier-Stokes equations in a tube structure. I. The case without boundary-layer-in-time. Nonlinear Anal. 122, 125-168 (2015)

[14] Panasenko, G., Pileckas, K.: Asymptotic analysis of the non-steady Navier-Stokes equations in a tube structure. II. General case. Nonlinear Anal. 125, 582-607 (2015) 
[15] Panasenko, G., Pileckas, K.: Divergence equation in thin-tube structures. Appl. Anal. 94(7), 1450-1459 (2015)

[16] Reynolds, O.: On the theory of lubrication and its application to MR. Beauchamp tower's experiments, including an experimental determination of the viscosity of olive oil. Philos Trans R Soc Lond 177, 157-234 (1886)

[17] Temam, R.: Navier-Stokes equations, volume 2 of Studies in Mathematics and its Applications. North-Holland Publishing Co., Amsterdam, revised edition, Theory and numerical analysis. With an appendix by F. Thomasset (1979)

A. Ghosh and V. A. Kozlov

Mathematics and Applied Mathematics, MAI

Linköping University

58183 Linköping

Sweden

e-mail: arpanghosh_isi@yahoo.co.in

V. A. Kozlov

e-mail: vladimir.kozlov@liu.se
S. A. Nazarov

St. Petersburg State University

Universitetsky pr., 28

Stary Peterhof

Russia 198504

e-mail: srgnazarov@yahoo.co.uk

and

Institute of Problems of Mechanical Engineering RAS

V.O., Bolshoj pr., 61

St. Petersburg

Russia 199178

(accepted: November 9, 2020; published online: February 25, 2021) 